\title{
Fundamental plane distances to early-type field galaxies in the South Equatorial Strip
}

\section{The spectroscopic data}

\author{
K.R. Müller ${ }^{12}$, G. Wegner ${ }^{1}$, S. Raychaudhury ${ }^{3}$, and W. Freudling ${ }^{2}$ \\ 1 Department of Physics and Astronomy, Dartmouth College, 6127 Wilder Laboratory, Hanover, NH 03755, U.S.A. \\ 2 European Southern Observatory and Space Telescope - European Coordinating Facility, Karl Schwarzschild Str. 2, D-85748 \\ Garching bei München, Germany \\ ${ }^{3}$ Inter-University Centre for Astronomy and Astrophysics, Ganeshkhind, Pune 411 007, India
}

Received June 25; accepted September 8, 1999

\begin{abstract}
Radial velocities and central velocity dispersions are derived for $238 \mathrm{E} / \mathrm{S} 0$ galaxies from mediumresolution spectroscopy. New spectroscopic data have been obtained as part of a study of the Fundamental Plane distances and peculiar motions of early-type galaxies in three selected directions of the South Equatorial Strip, undertaken in order to investigate the reality of largescale streaming motion; results of this study have been reported in Müller et al. (1998). The new APM South Equatorial Strip Catalog $(-17.5<\delta<+2.5)$ was used to select the sample of field galaxies in three directions: (1) $15 \mathrm{~h} 10$ - 16h10; (2) 20h30 - 21h50; (3) 00h10 - 01h30. The spectra obtained have a median $S / N$ per $\AA$ of 23 , an instrumental resolution (FWHM) of $\sim 4 \AA$, and the spectrograph resolution (dispersion) is $\sim 100 \mathrm{~km} \mathrm{~s}^{-1}$. The Fourier cross-correlation method was used to derive the radial velocities and velocity dispersions. The velocity dispersions have been corrected for the size of the aperture and for the galaxy effective radius. Comparisons of the derived radial velocities with data from the literature show that our values are accurate to $40 \mathrm{~km} \mathrm{~s}^{-1}$. A comparison with results from Jørgensen et al. (1995) shows that the derived central velocity dispersion have an rms scatter of 0.036 in $\log \sigma$. There is no offset relative to the velocity dispersions of Davies et al. (1987).
\end{abstract}

Key words: techniques: spectroscopic - galaxies: distances and redshifts - galaxies: elliptical and lenticular, cD - galaxies: fundamental parameters

Send offprint requests to: K.R. Müller

\section{Introduction}

The observed total radial velocity of a galaxy (found from the redshift) can be separated into two distinct parts: a cosmological part, from the expansion of the universe, and a peculiar motion associated with the galaxy proper motion. Measurements of galaxy peculiar velocities on large scales reveal the underlying mass density fluctuations, since galaxies will stream towards an overdense region and away from an underdense region.

To determine peculiar motions, a distance-indicator relation has to be used to find redshift-independent distances to galaxies. The Tully-Fisher relation can be used for spiral galaxies. Elliptical galaxies have been found to populate a nearly planar region in the three-dimensional space defined by the central velocity dispersion, the effective (half-luminosity) radius, and the effective surface brightness; this region is called the Fundamental Plane (Lucey et al. 1991; Jørgensen et al. 1993). The Fundamental Plane (FP) method for distance determination is an improvement on the $D_{n}-\sigma$ relation. It has a tighter correlation; therefore, a better precision in distances $(\sim 20 \%)$ can be achieved (Jørgensen et al. 1996; Scodeggio et al. 1997).

Galaxy peculiar velocities are found from a comparison of the distances with the measured redshifts. There is strong observational evidence for the existence of largescale flows in the local universe, induced by gravity (see Strauss \& Willick 1995). The dipole anisotropy of the cosmic microwave background $(\mathrm{CMB})$ radiation provides a natural velocity reference frame for the analysis of galaxy motions. The dipole anisotropy, determined from COBE, implies that the Local Group (LG) moves with respect to the CMB rest frame at $627 \pm 22 \mathrm{~km} \mathrm{~s}^{-1}$ towards $l=276 \pm 3^{\circ}, b=+30 \pm 3^{\circ}$ (Kogut et al. 1993). If this 
has a kinematic origin then, sufficiently far away, galaxy peculiar velocities should converge to the CMB frame.

Until now, the only studies which have reported measurements of the velocity field as far out as $15000 \mathrm{~km} \mathrm{~s}^{-1}$ are those of Lauer \& Postman (LP) (1994), using brightest cluster galaxies as distance indicators, and Riess et al. (1995), using Type Ia supernovae. LP checked the convergence of the LG dipole motion to the CMB dipole, with a surprising result: a strong signature of a very large-scale bulk flow was seen, with an amplitude of $689 \pm 178 \mathrm{~km} \mathrm{~s}^{-1}$ in the direction $l=343^{\circ}, b=+52^{\circ}$. The LP study implies that the local rest frame fails to converge to the $\mathrm{CMB}$ frame, even in regions with radii $\sim 15000 \mathrm{~km} \mathrm{~s}^{-1}$. A bulk flow with the statistical significance of this result rules out a whole series of cosmological models at the $>95 \%$ confidence level (Feldman \& Watkins 1994; Strauss et al. 1995); the LP result is in disagreement with all viable models at present.

The LP sample extended to $15000 \mathrm{~km} \mathrm{~s}^{-1}$, with an effective depth of $\sim 8000 \mathrm{~km} \mathrm{~s}^{-1}$. Therefore, the logical next step was to compare the LP result with peculiar velocities as found from applying the Tully-Fisher and FP methods to galaxies extending further out than any previous peculiar velocity studies. From Tully-Fisher studies of field and cluster spiral galaxies within $8000 \mathrm{~km} \mathrm{~s}^{-1}$, Giovanelli et al. $(1996,1998 \mathrm{a}, 1998 \mathrm{~b})$ concluded that these galaxies do not show any evidence of such a bulk flow.

In order to investigate the reality of large-scale streaming motion on scales of up to $150 \mathrm{Mpc}$, we have studied the peculiar motions of 179 early-type galaxies in three directions of the South Equatorial Strip, at distances out to $\sim 20000 \mathrm{~km} \mathrm{~s}^{-1}$. We have obtained new and independent measurements of the peculiar velocity field of elliptical field galaxies at a depth similar to that of LP, using a combination of photometric and spectroscopic data. For further details of the project, see Müller (1997); the results for peculiar motions are analysed in Müller et al. (1998).

In this paper we present the spectroscopic data used in our study of the large-scale motions. From the spectra, galaxy redshifts were measured, and central velocity dispersions were obtained - the accurate determination of these is essential for the FP to be applied as a distance indicator. This paper is organised as follows. Sample selection and observations are described in Sect. 2, the basic reduction of the spectra is covered in Sect. 3, and the radial velocities and central velocity dispersions are derived in Sect. 4. The applied corrections are discussed in Sect. 5, and the results are given in data tables in Sect. 6 . In Sect. 7 the results are compared internally and with results from the literature.

\section{Samples and observations}

Sample galaxies were selected from the new Automatic Plate Measuring Facility (APM) South Equatorial Strip
Catalog, which was made available prior to publication (Raychaudhury \& Lynden-Bell 2000). The South Equatorial Strip Catalog $(-17.5<\delta<+2.5)$ is an uncharted region in the velocity field, because previously no good galaxy catalog existed for this region, and consequently the peculiar motions of galaxies in this strip had never been mapped. Redshifts had been previously measured for only about 20 of the sample galaxies, and velocity dispersion data existed for only a few of the sample galaxies.

The sample consists of early-type galaxies in three selected directions: (1) 15h10-16h10; (2) 20h30-21h50; (3) 00h10-01h30. The first region is about $20^{\circ}$ from the direction of the LP bulk flow, the second region is almost perpendicular to the first, and the third is on the opposite side of the sky from the first, close to the direction of the Perseus-Pisces region and the South Galactic Pole. The APM South Equatorial Strip Catalog has a magnitude limit of $b_{j}=17.0 \mathrm{mag}$, which corresponds to $15.05 \mathrm{mag}$ in Kron-Cousins $R$. All candidate galaxies in the three regions were examined on the POSS plates and then on CCD images to verify the morphological type. The original intention was to observe all galaxies down to this magnitude limit. We have both photometric and spectroscopic data for 179 of these galaxies, which resulted in a sample of E/S0 galaxies virtually complete to $R=14.0$. The completeness drops for fainter magnitudes; galaxies down to $R=15.0$ are included. New observations were carried out for all 179 galaxies, so that the sample has a fully independent data set with homogeneous observations, uniform data reduction, and consistent measurement techniques for all the galaxies.

In addition to the galaxies in the three sample regions, a number of standard galaxies from previous studies were also observed, for comparison purposes, in order to confirm the accuracy of the spectroscopic parameters obtained in this work. Comparison galaxies were selected from the following samples in the literature: Davies et al. (1987); González (1993); McElroy (1995); and Jørgensen et al. (1995). Spectra were also obtained for a sample of 40 galaxies in the Coma cluster, which was used as the calibration cluster for the FP distance-indicator relation. Galaxies were chosen to be E/S0 using earlier studies (e.g. Lucey et al. 1991; Jørgensen et al. 1993), and most lie within about $0.5^{\circ}$ of the point midway between NGC 4889 and NGC 4874.

Spectroscopic observations were done at the $2.4 \mathrm{~m}$ Hiltner telescope and the $1.3 \mathrm{~m} \mathrm{McGraw-Hill} \mathrm{telescope}$ of the Michigan-Dartmouth-M.I.T. (MDM) Observatory on Kitt Peak, Arizona, and also at the $4.4 \mathrm{~m}$ Multiple Mirror Telescope ${ }^{1}$ (MMT) on Mount Hopkins, Arizona. Data were collected during a series of nine observing runs

1 The Multiple Mirror Telescope Observatory is operated by the Harvard-Smithsonian Center for Astrophysics jointly with the University of Arizona. 
Table 1. Details of spectroscopic observing runs

\begin{tabular}{lcccccc}
\hline $\begin{array}{l}\text { Run } \\
\text { Code }\end{array}$ & Dates of Observations & Telescope & $\begin{array}{c}\text { Wavelength } \\
\text { Range }(\AA)\end{array}$ & $\begin{array}{c}\Delta \lambda \\
(\AA / \mathrm{pix})\end{array}$ & $\begin{array}{c}\text { Res } \\
(\AA)\end{array}$ & $\begin{array}{c}\text { Res } \\
\left(\mathrm{km} \mathrm{s}^{-1}\right)\end{array}$ \\
\hline & & & & & & \\
S1 & $28 / 06 / 93-04 / 07 / 93$ & MDM 2.4 m & $4290-6780$ & 2.43 & 4.7 & 110 \\
S2 & $16 / 06 / 94-21 / 06 / 94$ & MDM 2.4 m & $4440-6730$ & 2.24 & 3.9 & 93 \\
S3 & $11 / 09 / 94-18 / 09 / 94$ & MDM 2.4 m & $4675-7035$ & 2.31 & 5.0 & 118 \\
S4 & $25 / 09 / 94-27 / 09 / 94$ & MMT 4.4 m & $5000-5970$ & 0.81 & 1.6 & 40 \\
S5 & $21 / 10 / 94-23 / 10 / 94$ & MDM 2.4 m & $4295-7165$ & 2.81 & 5.6 & 133 \\
S6 & $09 / 03 / 95-14 / 03 / 95$ & MDM 2.4 m & $4305-6540$ & 2.19 & 4.4 & 103 \\
S7 & $29 / 05 / 95$ & MDM 1.3 m & $4000-7100$ & 3.04 & 5.6 & 132 \\
S8 & $05 / 06 / 95-11 / 06 / 95$ & MDM 2.4 m & $4320-6565$ & 2.20 & 4.3 & 102 \\
S9 & $01 / 09 / 95-04 / 09 / 95$ & MDM 2.4 m & $4320-6565$ & 2.20 & 4.3 & 102 \\
& & & & & & \\
\hline
\end{tabular}

Table 2. CCD and instrument parameters for spectroscopy

\begin{tabular}{lllll}
\hline & \multicolumn{4}{c}{ Run Code } \\
\cline { 2 - 5 } Parameter & $\begin{array}{l}\text { S1, S2, S3, } \\
\text { S6, S8, S9 }\end{array}$ & & S5 & S7 \\
& MDM 2.4 m & MMT 4.4 m & MDM 2.4 m & MDM 1.3 m \\
& Mark III & Red Channel & Mark III & Mark III \\
Telescope & grism $(600)$ & grating $(1200)$ & grism $(600)$ & grism $(600)$ \\
Spectrograph & 5800 & 5750 & 5800 & 4600 \\
Grating/grism $(l / \mathrm{mm})$ & 1.68 & 1.00 & 1.68 & 2.17 \\
Blaze $(\AA)$ & Tek CCD & Loral CCD & Loral CCD & Loral CCD \\
Slit width $(\operatorname{arcsec})$ & $1024 \times 1024$ & $800 \times 1200$ & $2048 \times 2048$ & $2048 \times 2048$ \\
Detector & $1 \times 1$ & $2 \times 1$ & $2 \times 2$ & $2 \times 2$ \\
Format & 6.0 & 7.0 & 5.4 & 5.4 \\
Binning & 3.45 & 2.60 & 1.94 & 1.94 \\
Readout Noise $\left(\mathrm{e}^{-}\right)$ & 24 & 15 & 30 & 30 \\
Gain $\left(\mathrm{e}^{-} /\right.$ADU) & 0.777 & 0.300 & 0.971 & 1.794 \\
Pixel size $(\mu \mathrm{m})$ & & & & \\
Spatial scale $(\operatorname{arcsec} /$ pixel $)$ & & & & \\
& & & & \\
\hline
\end{tabular}

between June 1993 and September 1995. Over 260 galaxy spectra were obtained. In total, 38 nights were allocated for this project, and 25 of them were usable. The main details of the observing runs are summarized in Table 1. (The resolution in $\AA$ given in Table 1 is the instrumental resolution, found from the FWHM of arc lines, whereas the resolution in $\mathrm{km} \mathrm{s}^{-1}$ is the spectrograph resolution or instrumental dispersion.) The spectral range was chosen to cover the Mgb band (around $\lambda_{0}=5177 \AA$ ), the E-band $(5270 \AA)$ and the Fe I line $(5335 \AA)$. For some runs the $\mathrm{H} \beta(4861 \AA)$ and Na D (5895 $\AA$ ) features were also included. All observations were made with a long slit and a CCD detector. The setup and instrument parameters for all runs are shown in Table 2.
All observations on the two telescopes at MDM were made with f/7.5 and using the Mark III spectrograph, which consists of a grism, glass optics, and a CCD detector. Two different detectors were used with the MDM telescopes: the Tektronix TK1024A $1024^{2}$ CCD, which is a thinned, back-illuminated CCD with a pixel size of $24 \mu \mathrm{m}$, and the Loral $2048^{2} \mathrm{CCD}$, which is a thick, frontilluminated CCD with a $15 \mu \mathrm{m}$ pixel size. Both CCDs are good cosmetically and have low readout noise. For the observations at the MMT we used the Red Channel: a spectrograph consisting of a collimator, a folding flat, a grating, and a CCD detector. The spectrograph was used in the high-throughput long-slit mode with a slit 180 arcsec long. The detector used at the MMT was a Loral $800 \times 1200$ CCD binned by 2 pixels in the narrower spatial 
Table 3. Radial velocity standard stars and template stars

\begin{tabular}{llrr}
\hline Star & $\begin{array}{l}\text { Spectral } \\
\text { Type }\end{array}$ & $\begin{array}{r}S / N \\
\text { per } \AA\end{array}$ & $\begin{array}{r}\text { Radial Velocity } \\
\left(\mathrm{km} \mathrm{s}^{-1}\right)\end{array}$ \\
\hline & & & \\
HD 4388 & K3 III & 62 & -28.3 \\
HD 12029 & K2 III & 350 & +38.6 \\
HD 20893 & K3 III & 110 & +5.9 \\
HD 22072 & DG7 & 120 & +12.2 \\
HD 36003 & K0 & 106 & -58.2 \\
HD 38751 & G8 III & 220 & +15.7 \\
HD 51440 & K2 III & 117 & +27.1 \\
HD 64606 & G5 & 137 & +93.8 \\
HD 65934 & K0 & 130 & +35.0 \\
HD 72324 & G9 III & 68 & +75.2 \\
HD 73665 & K0 III & 120 & +36.9 \\
HD 74377 & K0 & 84 & -25.4 \\
HD 90861 & K2 III & 140 & +36.3 \\
HD 132737 & K0 III & 110 & -24.1 \\
HD 165195 & G5 & 50 & -0.2 \\
HD 171232 & G8 III & 142 & -35.9 \\
HD 172401 & - & 102 & -73.0 \\
HD 194071 & G8 III & 127 & -9.8 \\
HD 213947 & K4 III & 280 & +16.7 \\
HD 223094 & K5 III & 375 & +19.6 \\
& & & \\
\hline
\end{tabular}

direction (perpendicular to the dispersion). The CCD is very good cosmetically, with only a few traps.

The slit was usually oriented with the long axis running North-South. The instrumental resolution FWHM (in pixels and in $\AA$ ) was determined by fitting a Gaussian to measure the widths of lines in calibration lamp spectra and of night-sky lines. Each galaxy was observed with a sufficiently long exposure time to ensure a high enough signalto-noise ratio $(S / N \sim 20)$ to enable the central velocity dispersion, as well as the redshift of the galaxy, to be determined accurately from the spectrum. At the MDM $2.4 \mathrm{~m}$ telescope, the average integration time needed was $2400 \mathrm{~s}$; the integration times for individual galaxies ranged from $900 \mathrm{~s}$ to $2 \times 3600 \mathrm{~s}$ according to the magnitude and surface brightness of the galaxy and the observing conditions. At the MMT, the average integration time per galaxy was 1200 s. A total of 263 spectra were obtained, of 238 galaxies. For some sample galaxies, more than one spectrum was obtained; an observation was repeated in some cases to provide a way to check the accuracy. Spectra were also obtained for KPNO IIDS spectrophotometric flux standard stars (Strom 1979), for use in flux calibration of the spectra. The exposure time was usually $360 \mathrm{~s}$, and one flux standard star was observed per night.
In addition, several stars with known radial velocities were observed during each run. (Values of radial velocity for the standard stars were taken from Wilson 1953; Evans 1970; Abt \& Biggs 1972; and Barbier-Brossat \& Petit 1987.) These radial velocity standard stars were chosen to be of luminosity class III and in the range of spectral types G5 - K5. Most were K giants fainter than 7th magnitude. The observed stars are listed in Table 3 . The same set of stars served as spectral templates for the determination of the velocity dispersions of the galaxies and as radial velocity standards for finding the galaxy redshifts. All the standard stars were trailed up and down the slit so that the slit illumination would be uniform and more similar to that of a galaxy. The exposure time was normally $420 \mathrm{~s}$, which usually resulted in a very high $S / N$ ratio.

Before and after the spectrum of each galaxy or standard star, a comparison spectrum was taken for use during wavelength calibrations. At MDM, $\mathrm{Hg}+\mathrm{Ne}$ and Ne lamps were exposed for $0.2 \mathrm{~s}$, and at the MMT $\mathrm{He}+\mathrm{Ne}+\mathrm{Cu}$ + Ar lamps were exposed for $120 \mathrm{~s}$. Each night a series of bias frames was taken, as well as spectral flat field frames using an internal continuum flat lamp. Exposure times for flat fields were $30 \mathrm{~s}$ at MDM and $2 \mathrm{~s}$ at the MMT.

\section{Preliminary data reduction procedures}

Packages in the image processing system IRAF ${ }^{2}$ (Tody 1986) were used for the basic reduction of the spectra. The main preliminary reduction steps, which are described in more detail below, are the following: (i) bias subtraction and dividing the data by a flat field; (ii) mapping the wavelength as a function of row and column by using the comparison exposures; (iii) subtracting the sky spectrum; (iv) removing the cosmic-ray hits from the spectra; and (v) extracting one-dimensional spectra from a sum over the aperture.

The bias level for each frame was found from the overscan region. The bias frames were averaged and the residual bias level subtracted pixel-by-pixel from each image. From dark frames with exposures of $2400 \mathrm{~s}$, a level always much less than one count was found. Since the dark current is flat, no dark count corrections were made. The flat field frames were combined to their median value, by using noao.imred.ccdred.flatcombine. This result was then normalized by fitting a cubic spline to the continuum in the wavelength direction and dividing the flat field by this fit to obtain the response function. The rms variation in the resulting flattened response frames was typically less than $0.5 \%$. Each galaxy or star spectral frame was then divided by the response function.

\footnotetext{
${ }^{2}$ IRAF is distributed by National Optical Astronomy Observatories, which is operated by the Association of Universities for Research in Astronomy, Inc., under cooperative agreement with the National Science Foundation, U.S.A.
} 


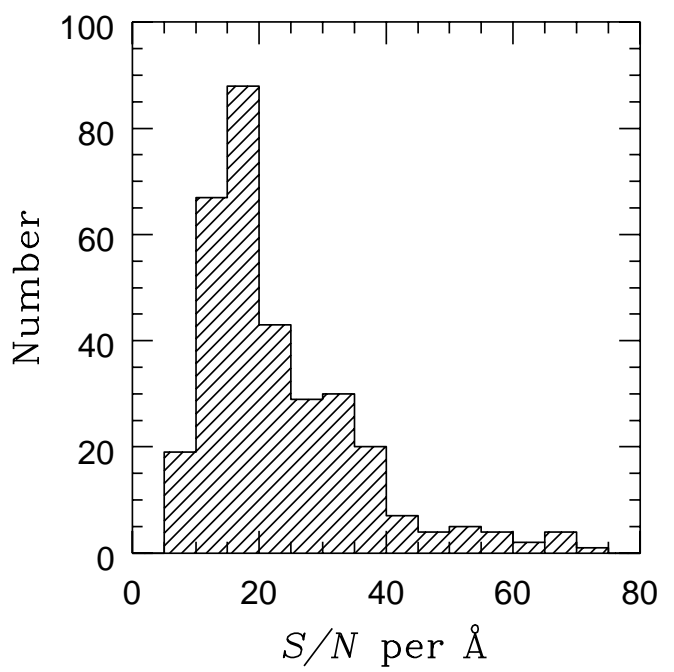

Fig. 1. The distribution of spectra as a function of the mean $S / N$ per $\AA$ measured at the wavelength of $\mathrm{Mgb}$

The next step was to transform from pixel coordinates to a two-dimensional spatial scale with wavelength coordinates along the dispersion axis of the CCD image. Tasks in the package noao.twodspec.longslit were used for this. By means of 25-50 identified arc lines in the comparison lamp spectra, a polynomial was fitted to the wavelength solution, with an rms residual in the coordinate fit of $0.1-0.2 \AA$. The two-dimensional spectra of stars and galaxies were calibrated in wavelength using the comparison lamp spectra observed before and after each object, and thus transformed to a linear wavelength scale.

The sky was fitted interactively using background, with the sky level found from unoccupied regions of the slit, and the sky background was subtracted. This worked well, since the galaxy occupied a relatively small part of the slit. Cosmic rays were removed by rejection using images. lineclean, with care being taken that absorption lines were unaffected. The final one-dimensional spectra were extracted by summing over an aperture covering the entire visible galaxy. This included tracing the mapping of the slit position across the CCD as a function of row or column. The package kpnoslit.apall was used interactively to fit the traced positions of the apertures. For all runs the trace was found to vary by at most 4 pixels across the CCD, in 1023 pixels. The typical width of the galaxy spectrum was about 20 pixels, so the maximum misalignment resulting from the tilt of the spectrum would be 0.1 pixels.

The summed one-dimensional spectra typically have $1000-20000$ counts at $5200 \AA$, near the wavelength of Mgb. The mean value of the $S / N$ per $\AA$ was found for each spectrum, of galaxies and standard stars. The $S / N$ was calculated from the mean number of photon counts in the spectrum in the wavelength range of the continuum bands of the Mgb spectral feature, also including in the noise the contribution of the readout noise and the effect of subtracting the sky spectrum. For most spectra the resulting value of $S / N$ per $\AA$ is in the range of $15-40$. A histogram of the frequency distribution of all the spectra with respect to $S / N$ is shown in Fig. 1. The mean $S / N$ for all spectra is 23.0. For sample galaxies it is 21.4, for Coma galaxies 27.3 , and for standard galaxies 26.8. The $S / N$ of a typical stellar observation is $100-200$, which means that the stellar spectra can be considered to be noiseless.

At this stage the one-dimensional extracted spectra were inspected. Usually the sky subtraction was reasonably accurate, but the spectra were checked for night-sky emission lines, particularly those of $[\mathrm{O}$ I] at $5577 \AA$ and $\mathrm{Hg}$ at $5461 \AA$, and if necessary these lines were removed by hand in cases of imperfect sky subtraction. There were sometimes cosmic rays which had not been removed completely, and these were also removed by hand. Three of the observed galaxies had spectra which were found to contain strong emission lines, and these galaxies were removed from the sample.

\section{Determination of redshifts and velocity dispersions}

The accurate determination of the line-of-sight central velocity dispersions of the galaxies is critical to FP analysis. A number of different methods exist for determining the velocity dispersion $\sigma$ and the radial velocity $c z$. These include the Fourier quotient method (Sargent et al. 1977), the Fourier cross-correlation method (Tonry \& Davis 1979), the Fourier difference method (Dressler 1979), and the Fourier fitting method (Franx et al. 1989).

For this study we used the Fourier cross-correlation method, as implemented in the IRAF package $r v$. fxcor which is based on the method of Tonry \& Davis (1979). The spectra of the galaxy and the stellar template are cross-correlated in Fourier space, and the resultant maximum peak is fitted by a smooth symmetric function. The width and pixel shift of the peak are measures of $\sigma$ and the galaxy redshift (in $\mathrm{km} \mathrm{s}^{-1}$ ), found by comparison with the known radial velocity of the template. An indicator of the accuracy of the resulting value of $\sigma$ is the $r$ value (Tonry \& Davis 1979).

All spectra were rebinned, using onedspec.dispcor, to linear logarithmic wavelength coordinates. Total flux was conserved, and the same dispersion parameters were used for all spectra from all runs, resulting in spectra with logarithmic wavelength bins of $\Delta \ln \lambda=6.4610^{-5}$. A cubic spline was fitted to the continuum for all spectra (template stars as well as galaxies). This fit was subtracted from the spectrum to flatten it; the resulting spectrum has zero mean in the continuum. The spectra were then Fourier filtered before the correlation. Data points outside the selected sample region were zeroed, and the ends of the region ( $12.5 \%$ on each end of the spectrum) were apodized with a cosine bell. A ramp function was used as 
the filter. The parameters of the filter were adjusted to find the best combination. After the galaxy and template spectra had been thus prepared, the two sets of spectra were cross-correlated. In most cases the best region for crosscorrelation was found to be $4900-5800 \AA$. This choice excludes the $\mathrm{H} \beta$ and $\mathrm{Na} \mathrm{D}$ lines.

The galaxy spectra were Fourier cross-correlated in fxcor against each standard star in turn. The observed FWHM of the cross-correlation peak was transformed into a value for $\sigma$ by direct calibration with broadened template spectra, using the preocedure outlined by Baggley (1996). The spectrum of each template star was convolved with Gaussians of various known widths in the range $0-700 \mathrm{~km} \mathrm{~s}^{-1}$, and the resulting broadened spectra were run in $\mathrm{fxcor}$ (with the same parameters) against the original template spectrum, giving the FWHM of the cross-correlation peak in each case. A calibration curve of this FWHM width versus the broadening $\sigma$ for Gaussians of different widths was produced for each template star observation, by linear interpolation between the FWHM values from fxcor. The galaxy FWHM values were then converted into values for $\sigma$ for the galaxy by reading off the calibration for that particular template star. An example of a calibration curve is shown in Fig. 2, for HD 194071, observed during run S8. In this case the resolution was approximately $100 \mathrm{~km} \mathrm{~s}^{-1}$, and it can be seen from the figure that below this value of $\sigma$ it is more difficult to find an accurate determination of velocity dispersion.

For every galaxy there is a set of different values for $\sigma$ and $c z$, each pair of values the result of running the galaxy against a different stellar template spectrum. The values obtained by Fourier cross-correlation show small systematic differences depending on which stellar template is used. Template stars from all runs were used, a total of 45 observations of 20 different stars (as listed in Table 3). The rms difference between the estimates for $c z$ and $\sigma$ from different template stars was typically $<1 \%$ in $c z$ and $\sim 4 \%$ in $\sigma$.

In Fig. 3 the mean $r$ value from the cross-correlation analysis of a spectrum is shown plotted against the $S / N$ of that spectrum. From this plot it can be seen that the scatter is larger than would be expected if the $S / N$ were the only factor affecting the $r$ value. It could be that $r$ is also sensitive to a mismatch between the features of the galaxy and those of some of the template spectra. Dalle Ore et al. (1991) found no systematic variation of the width of the cross-correlation curve with spectral type, and showed that errors in the velocity dispersion owing to spectral type mismatch are negligibly small. Jørgensen et al. (1995) found that template stars of the spectral type G8 - K3 result in significantly better fits than stars of types K4 - K5. For our sample data, we do not find this to be true in general. For a particular galaxy, certain templates work better than others, but we find that the best set of templates is different for each galaxy. We therefore combined the results for the velocity dispersion for each

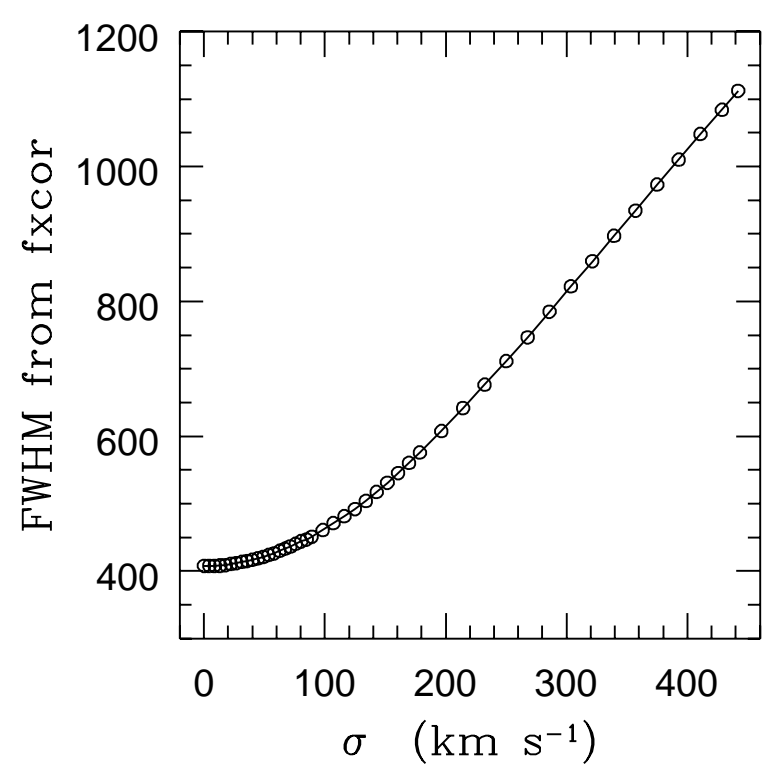

Fig. 2. The calibration curve for an observation of the standard star HD 194071. The points represent the FWHM from fxcor for the broadened template spectra compared with the unbroadened original template spectrum. The curve is found from linear interpolation between the points

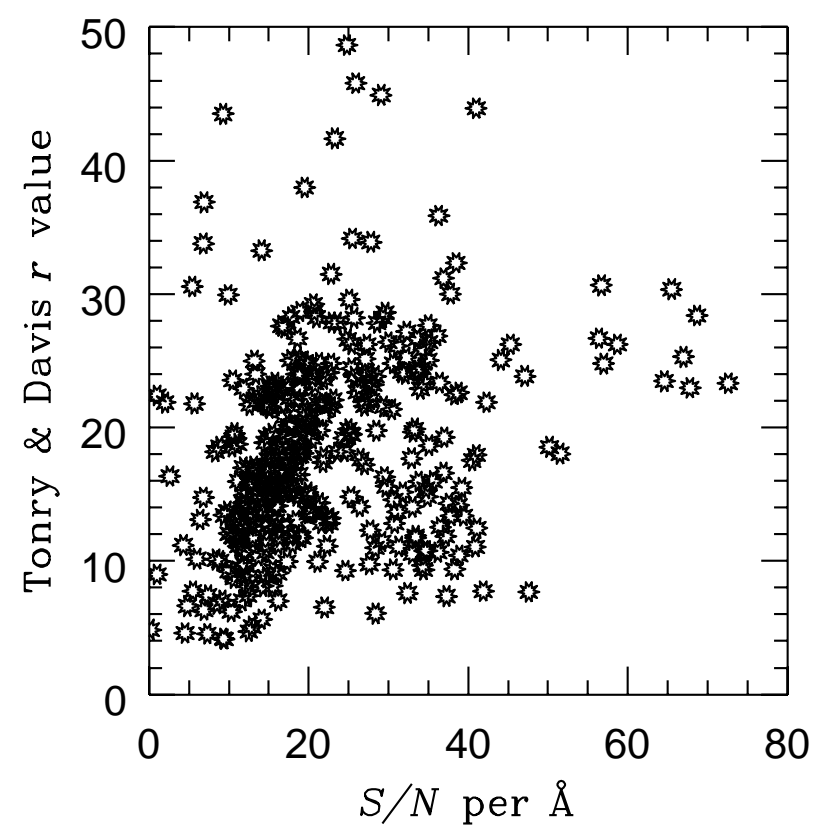

Fig. 3. A comparison of the $S / N$ per $\AA$ of the spectra with the value of the Tonry \& Davis $r$ parameter from the crosscorrelation, which is a measure of the ratio between the the peak height and the noise, and is therefore an indicator of the accuracy of the results 
galaxy in such a way as to minimize the effect of the template mismatch problem. First, the mean of all the results from different templates was taken. Then the $2 \sigma$ outliers were excluded and the mean was taken again, to give the final result. The redshift for each galaxy was estimated by calculating the mean observed heliocentric velocity from all the templates. For both $\sigma$ and $c z$, the standard deviations from the means were calculated.

To check the zero-point for the redshift determinations, the spectrum of each radial velocity standard star was cross-correlated with the spectra of all the other standard stars, resulting in estimates of relative velocity. The radial velocity standards were run against each other in fxcor using the same scripts with the same parameters as used for the galaxies. For each star the mean of the estimates of heliocentric redshift was found, and compared with the known value of radial velocity for that star. The rms difference between the mean estimated value and the known value was about $20 \mathrm{~km} \mathrm{~s}^{-1}$, which is therefore the accuracy of the zero-point of the radial velocities we determined.

Since a variation was observed among the separate determinations from each of the template spectra, estimates of the systematic errors for both redshift and velocity dispersion have been obtained from the rms scatter of the results from different templates. These error estimates are listed together with the results in Tables 5, 6, and 7 .

\section{Corrections to redshifts and velocity dispersions}

\subsection{Heliocentric correction to redshifts}

Redshifts were corrected for the radial velocity of the template star and also corrected to the heliocentric system to take into account the motion of the Earth relative to the observed galaxy and the template star. This correction is included in fxcor; the result in the heliocentric frame is denoted $v_{\text {hel }}$.

\subsection{Aperture correction to velocity dispersions}

In $\mathrm{E}$ and $\mathrm{S} 0$ galaxies there are radial gradients in the velocity dispersion, with a higher velocity dispersion in the center of the galaxy than in the outer regions (Davies \& Birkinshaw 1988; Franx et al. 1989; Davies et al. 1993). For this reason the derived "central" velocity dispersion parameter depends on the distance of the galaxy and on the size of the aperture used for the observation of the spectrum. It is therefore necessary to apply an aperture correction to transform the observed parameters so that they are independent of distance and of the telescope used.

The measured value of velocity dispersion depends on the velocity dispersion profile in the galaxy. Since the profile is not known for each galaxy, a general form must be assumed. Jørgensen et al. (1995) established an aperture correction from kinematic models based on the available literature data. They used the models to derive the equivalent circular aperture for each rectangular aperture, and adopted a power law as the aperture correction to $\sigma$. The radius $r_{\text {ap }}$ (in arcsec) of the equivalent circular aperture is found from $\left.r_{\text {ap }}=1.025 \sqrt{(} w l / \pi\right)$, where $w$ and $l$ are the width and length of a rectangular aperture (slit). Jørgensen et al. (1995) correct the velocity dispersion to an aperture with a standard physical size, and use a value for the normalising aperture size of $2 r_{\text {norm }}=1.19 h^{-1}$ $\mathrm{kpc}\left(h \equiv H_{0} / 100 \mathrm{~km} \mathrm{~s}^{-1} \mathrm{Mpc}^{-1}\right.$.) This means that the velocity dispersion values are normalized to a velocity dispersion measured with an aperture of diameter $1.19 \mathrm{~h}^{-1}$ $\mathrm{kpc}$, which is equivalent to 3.4 arcsec for a galaxy at the distance of the Coma cluster.

Baggley (1996) has shown that it is necessary to also take the effective radius $r_{\mathrm{e}}$ into account in the aperture correction, since the observed velocity dispersion of a galaxy depends on $r_{\mathrm{e}}$ as well. For two galaxies of different sizes at the same distance observed through the same aperture, the slit will cover more of the smaller galaxy and a different part of the galaxy profile will therefore be sampled; this dependence of $\sigma$ on $r_{\mathrm{e}}$ must be removed to ensure that the velocity dispersion is truly a distanceindependent quantity. We used Baggley's formula for the aperture correction; it is a generalisation of the formula of Jørgensen et al. (1995) to take $r_{\mathrm{e}}$ into account:

$\log \frac{\sigma_{\text {cor }}}{\sigma_{\text {obs }}}=0.038 \log \left(\frac{r_{\mathrm{ap}}}{r_{\text {norm }}} \frac{c z_{\text {gal }}}{c z_{\text {Coma }}} \frac{r_{\mathrm{e}}^{\text {norm }}}{r_{\mathrm{e}}^{\text {gal }}}\right)$,

where: $\sigma_{\text {obs }}$ is the value of the velocity dispersion found from observation through an aperture equivalent to $r_{\text {ap }}$; $\sigma_{\text {cor }}$ is the velocity dispersion value corrected to the adopted normalising aperture size $r_{\text {norm }} ; c z_{\text {gal }}$ is the redshift of the galaxy; $c z_{\text {Coma }}$ is the redshift of the Coma cluster; $r_{\mathrm{e}}^{\text {gal }}$ is the effective radius of the galaxy; and $r_{\mathrm{e}}^{\text {norm }}$ is the normalising effective radius, which is taken to be 20 arcsec, following Baggley (1996) - this is the mean $r_{\mathrm{e}}$ of the galaxies in the sample of Jørgensen et al. (1995), which was used to derive the correction. This means that there will be no aperture correction for a galaxy with $r_{\mathrm{e}}=20$ arcsec, at the distance of the Coma cluster, observed through an aperture with an equivalent radius of 1.7 arcsec.

Equation (1) was used to calculate the corrections. The slit widths in the various instrumental setups are as shown in Table 2. For the length of the slit in the aperture, what is important is the extent of the galaxy along the slit, i.e. the length from which the one-dimensional spectrum was extracted; the spectrum was extracted out to the point where the luminosity had fallen to $10 \%$ of its peak value. The $r_{\mathrm{e}}$ value for each galaxy was taken from the results of the fitting and seeing-correction programs applied to the photometric data, as described in Müller (1997) and Müller et al. (1999). The value of the heliocentric redshift 

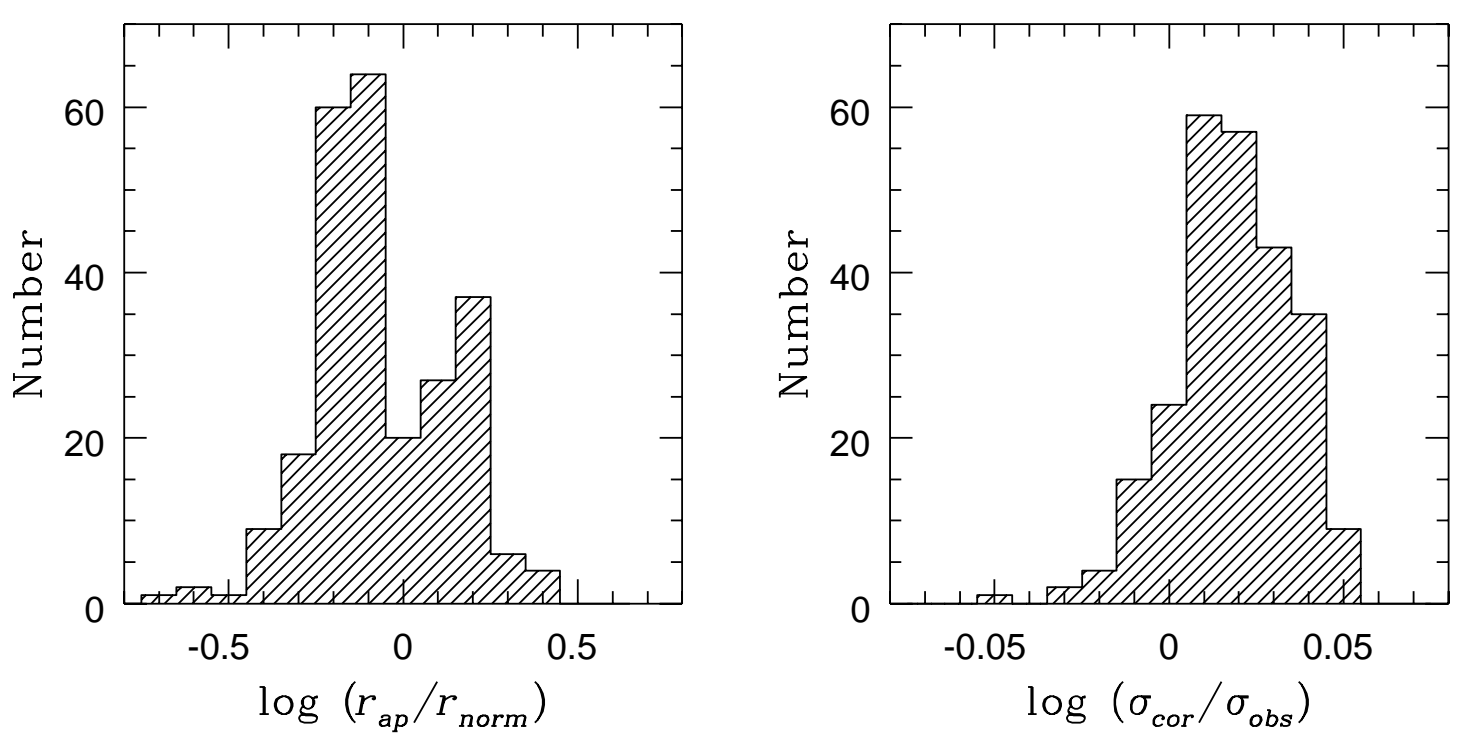

Fig. 4. Histograms for the galaxies in the three sample regions and in the Coma cluster. Left: A histogram of the aperture sizes of the sample galaxies relative to the normalising aperture radius, with $r_{\text {ap }}$ in units of $\mathrm{kpc}$ at the distance (redshift) of the galaxy, and $2 r_{\text {norm }}=1.19 h^{-1} \mathrm{kpc}$, equivalent to 3.4 arcsec for a galaxy at the distance of the Coma cluster ( $h=1$ was used). Right: A histogram of the sizes of the aperture corrections applied to the sample galaxies; $\sigma_{\text {obs }}$ is the raw observed value and $\sigma_{\text {cor }}$ is the aperture-corrected value of the velocity dispersion
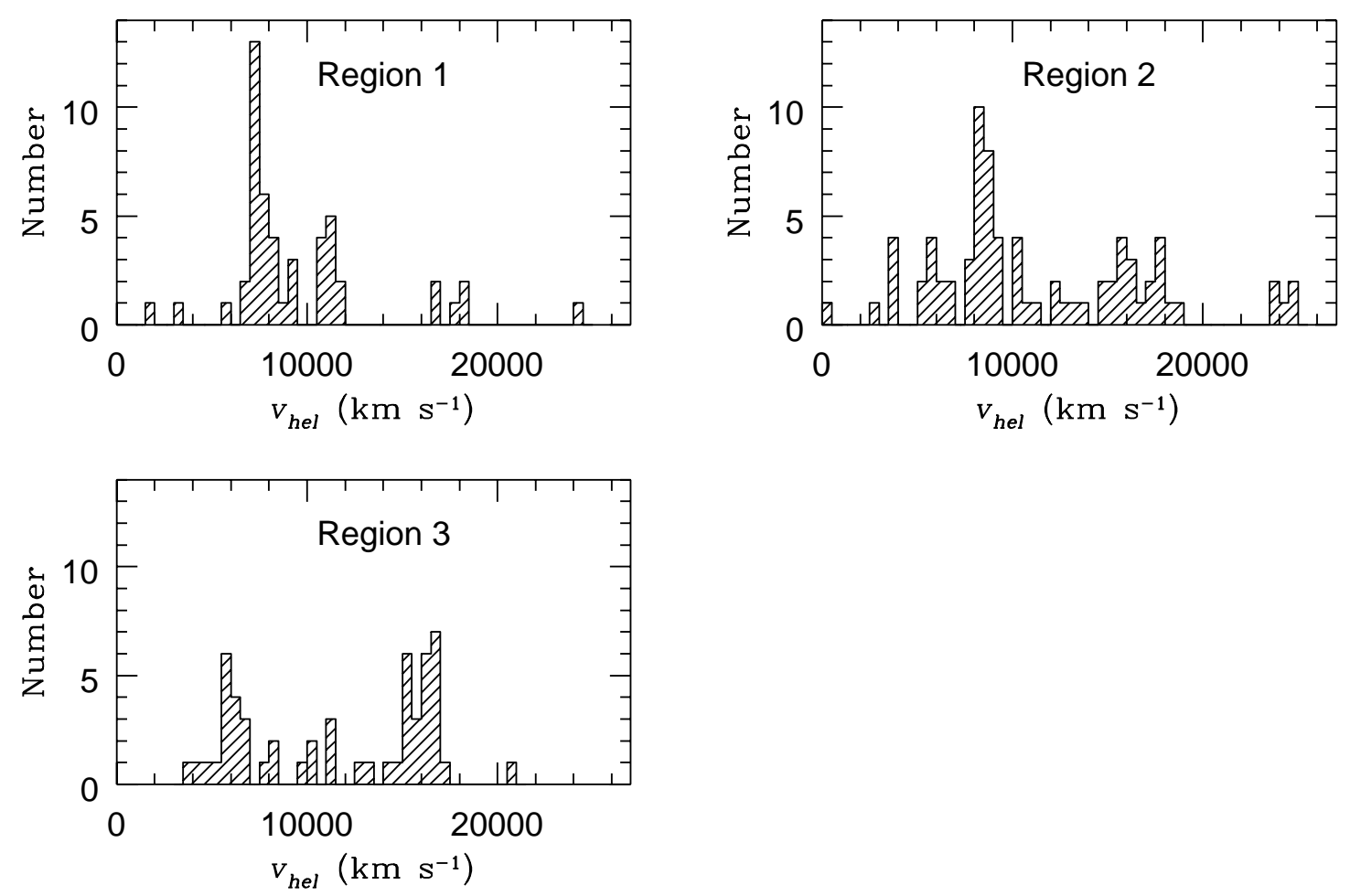

Fig. 5. Histograms of heliocentric radial velocity for observed galaxies in the three sample regions. Bin width $500 \mathrm{~km} \mathrm{~s} \mathrm{~s}^{-1}$ 


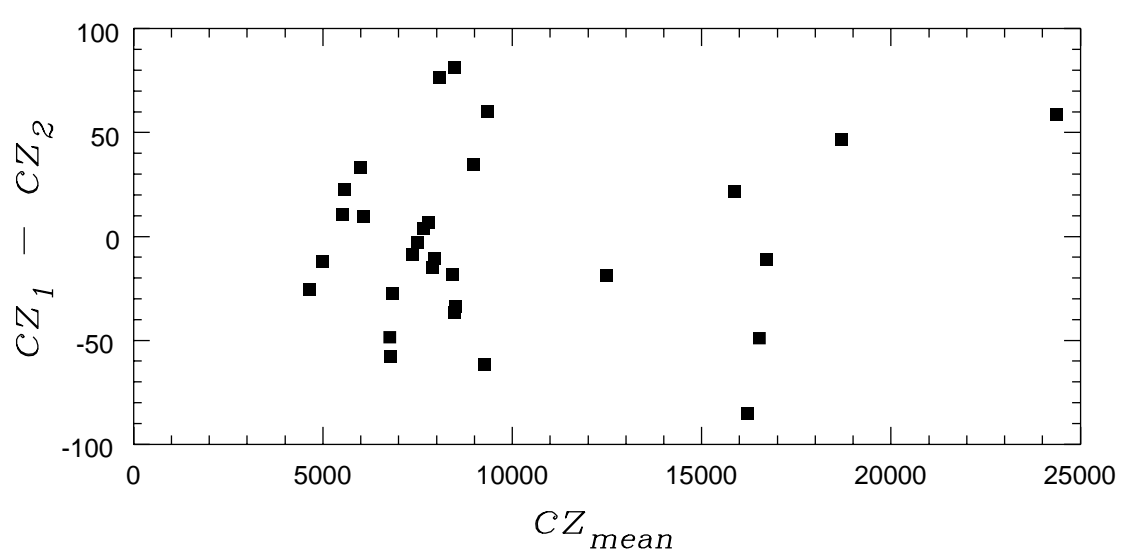

Fig. 6. Comparison of results for heliocentric radial velocity values determined from repeat observations
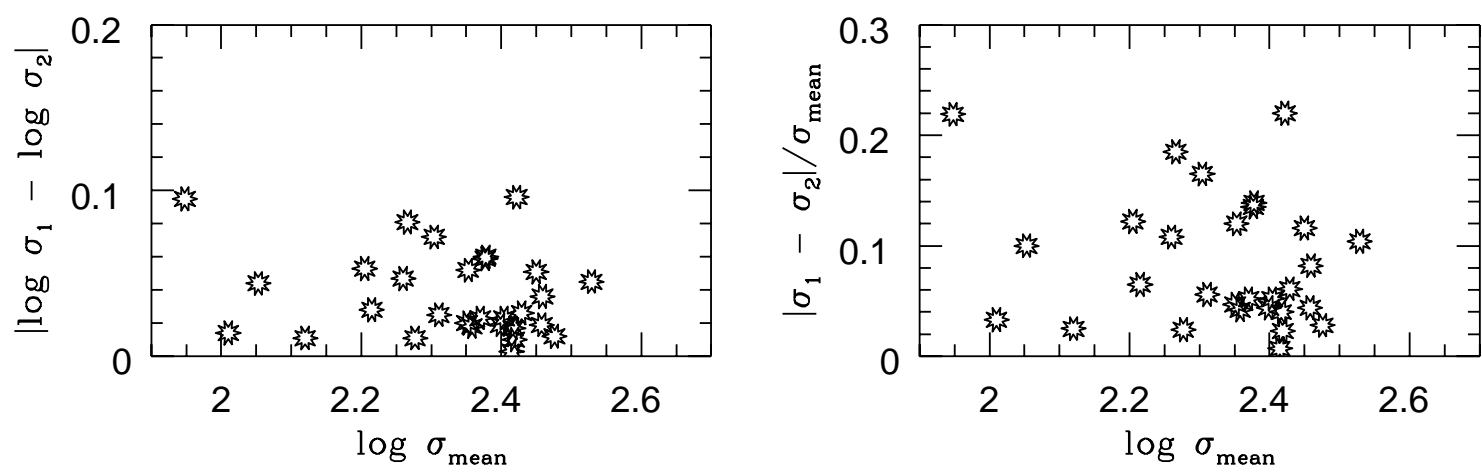

Fig. 7. Comparison of results for velocity dispersions measured from repeat observations

$c z_{\text {Coma }}$ was taken to be $6917 \mathrm{~km} \mathrm{~s}^{-1}$ (Zabludoff et al. 1993), and $h=1$ was used in the conversion of $\mathrm{km} \mathrm{s}^{-1}$ to kpc.

The contribution to the aperture correction from the effect of $r_{\mathrm{e}}$ is more important than those for different slit sizes and different galaxy distances. From Eq. (1) it can be seen that the correction is negative for large or nearby objects (this is the case for the standard galaxies) and positive for galaxies with $r_{\mathrm{e}}$ less than 20 arcsec, which are smaller than or more distant than a 20 arcsec galaxy at the distance of Coma. The histogram of the applied aperture corrections in Fig. 4 shows that for the sample galaxies the correction is positive in most cases.

\section{Results}

\subsection{Quality ratings for spectroscopic parameters}

A quality rating was assigned to the results from each spectrum, on the basis of the cross-correlation results, and also after visual inspections of the spectra. Quality ratings were made as follows: $Q=1$ for excellent spectra $(S / N$ per $\AA>20$; mean $S / N \sim 30) ; Q=2$ for good spectra (mean $S / N \sim 16$ ); $Q=3$ for acceptable spectra (mean

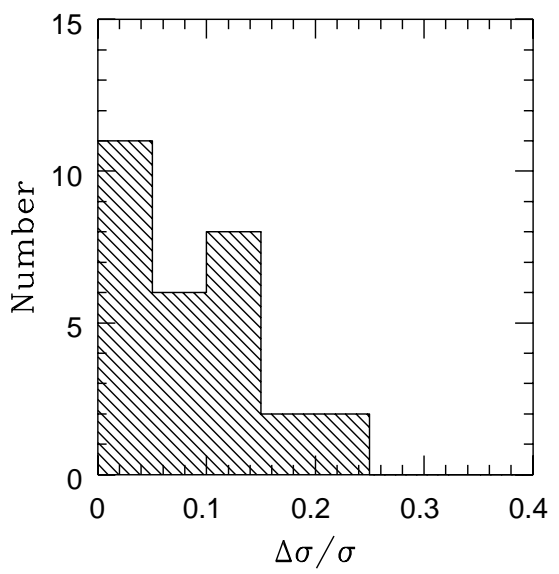

Fig. 9. Histogram of the fractional errors in the values of $\sigma$ determined from repeat observations

$S / N \sim 11)$; and $Q=4$ for poor spectra $(S / N<10$; mean $S / N \sim 4)$. The mean Tonry \& Davis $r$ values for the four quality ratings are $\sim 23, \sim 17, \sim 13$, and $\sim 7$ for ratings of $Q_{\mathrm{spec}}=1,2,3$, and 4 respectively. The percentage distribution of the quality ratings is shown in Table 4. 


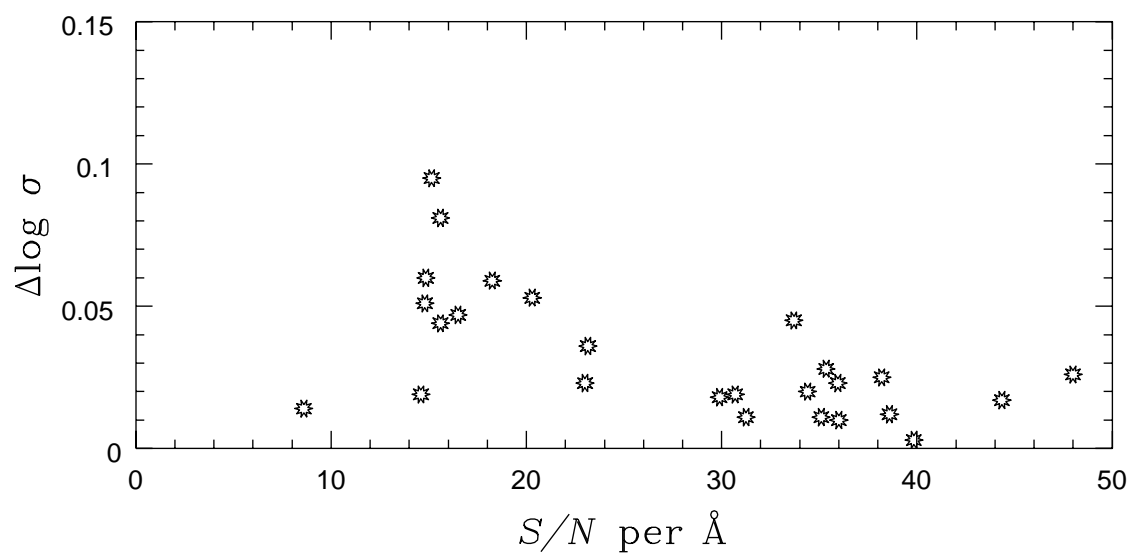

Fig. 8. The difference between results for velocity dispersions measured from repeat observations, plotted against the mean $S / N$ per $\AA$ of each pair of spectra

Table 4. Percentage distribution of quality ratings

Quality Rating $\quad$ Percentage of Sample Galaxies

Standard galaxies $\quad$ Region $1 \quad$ Region 2 Region $3 \quad$ Coma galaxies

\begin{tabular}{rrrrrr}
\hline$Q_{\text {spec }}=1$ & 72 & 24 & 62 & 63 & 43 \\
$Q_{\text {spec }}=2$ & 28 & 26 & 26 & 28 & 32 \\
$Q_{\text {spec }}=3$ & 0 & 46 & 10 & 9 & 25 \\
$Q_{\text {spec }}=4$ & 0 & 4 & 1 & 0 & 0 \\
\hline
\end{tabular}

For the spectra in the three sample regions, $78 \%$ of the spectra are of quality 1 or 2 .

The $S / N$ required to obtain a good measurement of radial velocity is lower than the $S / N$ required to obtain a reliable velocity dispersion. For the galaxies with $Q=4$ the signal was not strong enough to enable a reliable determination of the velocity dispersion. This was the case for galaxies of low surface brightness for which it was difficult to obtain spectra with a high enough $S / N$ ratio. From these weak spectra, only redshifts were found, and these galaxies were not included in the sample used for distance determination. Below a certain limit, the values of velocity dispersion are less reliable. In the final sample we used only galaxies with high enough $\sigma$ values to be determined accurately. The lower limit on usable $\sigma$ values depends on the instrumental resolution. For the spectra taken at MDM, with a dispersion of $\sim 2.3 \AA /$ pix and an instrumental resolution of $\sim 130 \mathrm{~km} \mathrm{~s}^{-1}$, only velocity dispersions which are larger than $130 \mathrm{~km} \mathrm{~s}^{-1}$ can be measured reliably. For the spectra from the MMT, for which the dispersion was $\sim 0.80 \AA /$ pix and the instrumental resolution was $\sim 40 \mathrm{~km} \mathrm{~s}^{-1}$, the limit is lower.

\subsection{Results for spectroscopic parameters}

The results for the spectroscopic parameters are presented in Tables 5, 6, and 7. In cases where there were two or more spectra of the same galaxy available, the results with the smallest errors, from the spectrum with the highest $S / N$, were used. Histograms of the redshift distributions in the three sample regions are plotted in Fig. 5.

Spectroscopic results for the sample galaxies are given in Table 5. The columns in the table are as follows:

1. Galaxy number in the APM catalog (or the galaxy name from the NGC or CGCG catalog in a few cases); the first three digits of the APM galaxy number are the UK Schmidt plate number.

2 \& 3. Right Ascension and Declination (epoch J1950.0) taken from the APM catalog.

$4 \& 5$. Magnitude $b_{J}$ (in mag) and major-axis diameter $d$ (in arcmin) from the APM catalog (both values corrected for Galactic extinction). Preliminary values prior to final calibrations.

6. Heliocentric redshift $v_{\text {hel }}$ (in $\left.\mathrm{km} \mathrm{s}^{-1}\right)$.

7. Estimated internal error in $v_{\text {hel }}\left(\right.$ in $\left.\mathrm{km} \mathrm{s}^{-1}\right)$.

8. Central velocity dispersion $\sigma$ (in $\mathrm{km} \mathrm{s}^{-1}$ ). 
Table 5. Spectroscopic parameters for the sample galaxies

\begin{tabular}{|c|c|c|c|c|c|c|c|c|c|}
\hline Galaxy & Right Ascension & Declination & $b_{J}$ & $d$ & $v_{\text {hel }}$ & $\operatorname{err}_{\mathrm{v}}$ & $\sigma$ & $\operatorname{err}_{\sigma}$ & $Q_{\text {spec }}$ \\
\hline \multicolumn{10}{|l|}{ Region 1} \\
\hline 87101144 & 160152.14 & -015905.0 & 13.9 & 1.3 & 9259 & 26 & 305 & 4 & 1 \\
\hline 65301306 & $1516 \quad 17.18$ & -154854.6 & 14.3 & 1.2 & 7296 & 17 & 270 & 16 & 1 \\
\hline 65300653 & 152414.86 & -124829.1 & 14.3 & 1.2 & 7351 & 15 & 232 & 21 & 1 \\
\hline 72501710 & 151751.60 & -082100.2 & 14.4 & 1.3 & 7573 & 22 & 149 & 18 & 3 \\
\hline 79800188 & $1547 \quad 10.58$ & -071944.2 & 14.4 & 1.3 & 8032 & 28 & 208 & 3 & 1 \\
\hline 65301588 & 151223.45 & -142557.7 & 14.5 & 1.1 & 7419 & 18 & 286 & 5 & 3 \\
\hline 65301596 & 151214.43 & -142339.5 & 14.5 & 1.0 & 7475 & 18 & 168 & 9 & 1 \\
\hline 87001759 & 153617.64 & -013419.3 & 14.6 & 1.3 & 3439 & 4 & 154 & 3 & 1 \\
\hline 65401564 & 154157.11 & -151830.0 & 14.8 & 1.1 & 7320 & 19 & 209 & 15 & 1 \\
\hline 72700354 & 160748.41 & -114345.9 & 14.8 & 0.9 & 7964 & 17 & 109 & 25 & 3 \\
\hline 79800136 & 154802.65 & -072436.0 & 14.9 & 0.8 & 7742 & 10 & 231 & 13 & 3 \\
\hline $87101109 \mathrm{E}$ & 160153.82 & -013641.8 & - & - & 9069 & 21 & 313 & 1 & 2 \\
\hline 65402690 & 153432.77 & -162559.2 & 15.0 & 1.0 & 7196 & 18 & 291 & 19 & 1 \\
\hline CGCG2158 & 151302.87 & +022556.5 & 15.0 & 0.6 & 1849 & 22 & 63 & 17 & 1 \\
\hline 72601433 & 153140.06 & -084421.2 & 15.0 & 0.8 & 7129 & 17 & 265 & 13 & 1 \\
\hline 79701386 & 152015.85 & -070910.5 & 15.1 & 1.2 & 11730 & 17 & 239 & 5 & 2 \\
\hline 65402668 & 153440.55 & -162536.1 & 15.1 & 0.7 & 6798 & 12 & 147 & 13 & 1 \\
\hline 79702357 & 151255.64 & -053558.1 & 15.1 & 1.2 & 11404 & 29 & 228 & 2 & 2 \\
\hline 79801072 & 153047.53 & -045545.2 & 15.1 & 0.7 & 8000 & 15 & 171 & 11 & 3 \\
\hline 87000696 & 154459.18 & -012330.8 & 15.1 & 0.7 & 8972 & 20 & 276 & 17 & 2 \\
\hline 65401595 & 154143.20 & -151544.8 & 15.1 & 0.8 & 7439 & 14 & 175 & 9 & 2 \\
\hline 79700187 & 152943.12 & -063546.2 & 15.2 & 0.9 & 11112 & 8 & 240 & 8 & 3 \\
\hline 65403060 & 153144.41 & -162753.1 & 15.2 & 1.1 & 7403 & 15 & 106 & 8 & 3 \\
\hline 65300973 & 152029.81 & -154934.3 & 15.3 & 0.8 & 7109 & 19 & 130 & 7 & 3 \\
\hline 65501546 & 155850.53 & -152219.0 & 15.3 & 0.9 & 7510 & 11 & 171 & 10 & 3 \\
\hline 65402909 & 153254.07 & -163702.4 & 15.3 & 0.9 & 6849 & 13 & 142 & 15 & 3 \\
\hline 87002300 & 153024.64 & +003631.6 & 15.3 & 0.8 & 10980 & 18 & 248 & 17 & 3 \\
\hline 65401438 & 154248.68 & -165232.8 & 15.4 & 0.8 & 11342 & 28 & 249 & 11 & 2 \\
\hline 65300169 & 152948.37 & -172603.3 & 15.4 & 0.7 & 7050 & 6 & 131 & 8 & 1 \\
\hline 65401086 & 154506.95 & -131413.8 & 15.4 & 0.9 & 11275 & 13 & 184 & 14 & 3 \\
\hline 65402811 & 153340.66 & -160632.6 & 15.5 & 0.6 & 7855 & 10 & 206 & 8 & 2 \\
\hline 72600774 & 154056.84 & -085911.0 & 15.5 & 0.6 & 17737 & 22 & 316 & 9 & 3 \\
\hline 87002308 & 153019.55 & +0038 12.6 & 15.6 & 0.7 & 11781 & 22 & 323 & 15 & 2 \\
\hline 79901311 & 155733.40 & -064640.9 & 15.6 & 0.7 & 9146 & 18 & 98 & 12 & 3 \\
\hline 79800922 & 153330.16 & -051158.9 & 15.6 & 0.6 & 7077 & 28 & 127 & 5 & 3 \\
\hline 65301134 & 151821.41 & -133145.3 & 15.6 & 0.6 & 7263 & 13 & 163 & 15 & 2 \\
\hline 72502368 & 151233.13 & -121326.2 & 15.7 & 0.7 & 7634 & 36 & 82 & 4 & 3 \\
\hline 65502352 & 155205.13 & -171043.8 & 15.7 & 0.9 & 16533 & 11 & 229 & 12 & 3 \\
\hline 65402361 & 153632.47 & -163216.3 & 15.7 & 0.6 & 10505 & 31 & 232 & 8 & 2 \\
\hline 72502210 & 151349.98 & -084951.2 & 15.7 & 0.7 & 5600 & 13 & 16 & 16 & 3 \\
\hline 87001111 & $1541 \quad 10.37$ & -013546.1 & 15.8 & 0.6 & 16897 & 20 & 223 & 6 & 3 \\
\hline 72600488 & 154454.15 & -114303.4 & 15.8 & 0.7 & 24012 & 51 & 397 & 62 & 3 \\
\hline 87100850 & 160428.03 & -014133.1 & 15.9 & 0.7 & 10839 & 10 & 132 & 22 & 3 \\
\hline 79702517 & 151138.51 & -041450.8 & 15.9 & 0.8 & 10978 & 23 & 413 & 11 & 2 \\
\hline 79800965 & 153246.84 & -031902.3 & 15.9 & 0.5 & 18012 & 19 & 303 & 8 & 3 \\
\hline 65502359 & 155207.79 & -151322.9 & 15.9 & 0.6 & 17513 & 44 & - & - & 4 \\
\hline 87002295 & 153027.12 & +010812.7 & 16.1 & 0.6 & 15068 & 30 & - & - & 4 \\
\hline 86902718 & 151028.74 & +021243.1 & 16.1 & 0.5 & 11391 & 32 & 177 & 22 & 3 \\
\hline 72801203 & 161751.62 & -102059.9 & 14.6 & 1.1 & 8472 & 29 & 215 & 4 & 2 \\
\hline 72801189 & 161757.70 & -094617.2 & 14.9 & 0.9 & 8006 & 24 & 209 & 2 & 2 \\
\hline
\end{tabular}


Table 5. continued

\begin{tabular}{|c|c|c|c|c|c|c|c|c|c|}
\hline Galaxy & Right Ascension & Declination & $b_{J}$ & $d$ & $v_{\text {hel }}$ & $\operatorname{err}_{\mathrm{v}}$ & $\sigma$ & $\operatorname{err}_{\sigma}$ & $Q_{\text {spec }}$ \\
\hline \multicolumn{10}{|l|}{ Region 2} \\
\hline NGC 7010 & 210155.45 & $-1232 \quad 17.1$ & 14.3 & 1.9 & 8480 & 19 & 228 & 3 & 1 \\
\hline 81405781 & 205356.12 & -040120.4 & 14.7 & 1.1 & 6025 & 10 & 156 & 10 & 1 \\
\hline 81303405 & 203510.59 & -061600.6 & 15.1 & 1.0 & 3844 & 5 & 89 & 5 & 1 \\
\hline 81502978 & 211738.08 & -050520.4 & 15.1 & 0.9 & 9102 & 17 & 367 & 9 & 3 \\
\hline 67200327 & 214453.58 & -132453.2 & 15.1 & 0.8 & 5418 & 13 & 167 & 14 & 1 \\
\hline 81303115 & 203622.29 & -050859.3 & 15.2 & 1.2 & 3797 & 10 & 131 & 19 & 1 \\
\hline 74402521 & 214816.53 & -093002.4 & 15.2 & 0.9 & 5380 & 20 & 213 & 15 & 1 \\
\hline 67003337 & 205246.63 & -133934.8 & 15.3 & 0.9 & 24515 & 38 & 555 & 7 & 3 \\
\hline 66901323 & 203901.84 & -140130.9 & 15.4 & 0.8 & 8409 & 18 & 265 & 2 & 1 \\
\hline 81603636 & 213327.73 & -033344.1 & 15.4 & 0.8 & 8027 & 14 & 257 & 11 & 3 \\
\hline 74402642 & 214650.74 & -100544.3 & 15.5 & 1.1 & 23869 & 10 & 598 & 14 & 2 \\
\hline 81405745 & 205359.57 & -033227.4 & 15.5 & 0.6 & 5979 & 11 & 126 & 14 & 1 \\
\hline 67001814 & 210046.20 & -151030.3 & 15.5 & 1.1 & 8455 & 16 & 241 & 13 & 1 \\
\hline 81503740 & 211412.52 & -044224.4 & 15.6 & 0.8 & 13765 & 11 & 266 & 22 & 1 \\
\hline 67003179 & 205339.80 & -143543.1 & 15.6 & 0.8 & 8468 & 6 & 139 & 3 & 2 \\
\hline 81601185 & 214501.36 & -063543.1 & 15.6 & 0.9 & 6103 & 15 & 153 & 13 & 1 \\
\hline 81602395 & 213915.52 & -065613.7 & 15.6 & 0.8 & 6906 & 20 & 158 & 6 & 2 \\
\hline 81404709 & 205646.08 & -043808.9 & 15.7 & 0.8 & 4680 & 49 & - & - & 4 \\
\hline 67003696 & 205048.81 & -144158.9 & 15.7 & 0.8 & 7580 & 11 & 178 & 6 & 1 \\
\hline 81602330 & 213929.90 & -042637.3 & 15.7 & 0.7 & 8225 & 25 & 114 & 8 & 1 \\
\hline 67102806 & 212746.38 & -162028.2 & 15.7 & 0.7 & 9353 & 7 & 215 & 4 & 1 \\
\hline 74400983 & $2141 \quad 10.20$ & -081811.9 & 15.7 & 0.9 & 15305 & 22 & 351 & 4 & 1 \\
\hline 81404583 & 205705.76 & -025034.2 & 15.8 & 0.8 & 17209 & 34 & 510 & 16 & 2 \\
\hline 74401483 & 213555.30 & -080758.1 & 15.8 & 0.9 & 15751 & 7 & 348 & 3 & 2 \\
\hline 67201749 & 213730.96 & -142730.1 & 15.8 & 0.6 & 18358 & 25 & 514 & 13 & 1 \\
\hline 67202822 & 213818.48 & -165516.6 & 15.8 & 0.7 & 15509 & 29 & 469 & 13 & 1 \\
\hline 67103161 & 212015.95 & -161158.7 & 15.8 & 0.7 & 13294 & 19 & 231 & 4 & 1 \\
\hline 67202363 & 214730.24 & -172435.4 & 15.8 & 0.6 & 10185 & 10 & 174 & 14 & 1 \\
\hline 74300882 & 211530.52 & -073202.2 & 15.8 & 0.7 & 8270 & 10 & 122 & 18 & 2 \\
\hline 67001913 & 210017.18 & -165957.9 & 15.9 & 0.6 & 8716 & 17 & 246 & 1 & 2 \\
\hline 81603664 & 213317.98 & -032220.5 & 15.9 & 0.6 & 2873 & 4 & 104 & 3 & 1 \\
\hline 81301006 & 204607.02 & -071050.8 & 15.9 & 0.7 & 12441 & 17 & 162 & 15 & 1 \\
\hline 67001942 & 210005.85 & -160034.5 & 15.9 & 0.7 & 8501 & 27 & 341 & 7 & 1 \\
\hline 81602522 & 213830.84 & -043125.2 & 15.9 & 0.8 & 16219 & 11 & 268 & 14 & 1 \\
\hline 81302133 & 204042.55 & -044054.1 & 16.0 & 0.7 & 8611 & 19 & 140 & 13 & 3 \\
\hline 74301907 & 211933.98 & -093845.1 & 16.0 & 0.7 & 12340 & 14 & 297 & 11 & 1 \\
\hline 74200901 & 210507.05 & -103638.4 & 16.0 & 0.7 & 8780 & 19 & 213 & 5 & 1 \\
\hline 74405800 & 213426.16 & -112724.7 & 16.0 & 0.7 & 17794 & 21 & 266 & 11 & 1 \\
\hline 81601843 & 214157.89 & -06 2311.7 & 16.0 & 0.8 & 15768 & 31 & 249 & 1 & 3 \\
\hline 81601806 & 214205.59 & $-07 \quad 17 \quad 17.9$ & 16.0 & 0.7 & 5998 & 9 & 121 & 15 & 2 \\
\hline 67002128 & 205913.51 & -132213.3 & 16.0 & 0.7 & 8492 & 17 & 197 & 9 & 2 \\
\hline 81501871 & 212242.66 & -031932.6 & 16.0 & 0.6 & 5640 & 20 & 77 & 15 & 2 \\
\hline 81601380 & 214405.41 & -052738.9 & 16.0 & 0.7 & 6584 & 15 & 92 & 11 & 1 \\
\hline 74200951 & 210447.84 & -105506.0 & 16.0 & 0.7 & 8269 & 10 & 141 & 12 & 2 \\
\hline 81504194 & $21 \quad 1207.77$ & -023355.1 & 16.1 & 0.7 & 7957 & 7 & 111 & 3 & 2 \\
\hline 67002172 & 205859.41 & -133036.4 & 16.1 & 0.7 & 8822 & 19 & 254 & 7 & 1 \\
\hline 81603573 & 213347.15 & -041151.4 & 16.1 & 0.6 & 15726 & 30 & 195 & 2 & 2 \\
\hline 81602191 & 214010.46 & -070552.5 & 16.1 & 0.6 & 16043 & 33 & 321 & 1 & 2 \\
\hline 74401426 & 213627.77 & -073139.3 & 16.1 & 0.7 & 10425 & 21 & 49 & 24 & 2 \\
\hline 81602252 & 213951.84 & -063924.5 & 16.2 & 0.6 & 10348 & 30 & 134 & 6 & 2 \\
\hline
\end{tabular}


Table 5. continued

\begin{tabular}{|c|c|c|c|c|c|c|c|c|c|}
\hline Galaxy & Right Ascension & Declination & $b_{J}$ & $d$ & $v_{\text {hel }}$ & $\operatorname{err}_{\mathrm{v}}$ & $\sigma$ & $\operatorname{err}_{\sigma}$ & $Q_{\text {spec }}$ \\
\hline 81600601 & 214754.17 & -053633.7 & 16.2 & 0.6 & 16715 & 17 & 325 & 19 & 2 \\
\hline 67003806 & 205017.86 & -163130.7 & 16.2 & 0.6 & 12891 & 21 & 330 & 5 & 1 \\
\hline 81602134 & 214027.29 & -071752.8 & 16.2 & 0.6 & 15015 & 31 & 251 & 2 & 2 \\
\hline 74202489 & 205531.78 & -075712.4 & 16.2 & 0.8 & 23717 & 30 & 349 & 6 & 3 \\
\hline 67201815 & 213619.16 & -143525.1 & 16.3 & 0.6 & 17930 & 23 & 446 & 16 & 2 \\
\hline 67201810 & 213628.63 & -143241.1 & 16.3 & 0.5 & 17972 & 16 & 286 & 2 & 2 \\
\hline 81600354 & 214907.77 & -031357.5 & 16.4 & 0.6 & 16461 & 17 & 228 & 13 & 1 \\
\hline 74404851 & 214519.53 & -120859.7 & 16.4 & 0.7 & 14761 & 22 & 175 & 7 & 1 \\
\hline 81304119 & 203156.30 & -064017.5 & 16.4 & 0.6 & 24394 & 37 & 360 & 11 & 1 \\
\hline 67001459 & 210245.64 & -123459.3 & 16.4 & 0.5 & 7940 & 16 & 128 & 10 & 1 \\
\hline 67001377 & 210314.05 & -160059.9 & 16.4 & 0.6 & 8511 & 19 & 134 & 8 & 1 \\
\hline 67002239 & 205837.77 & -131752.6 & 16.4 & 0.6 & 9258 & 12 & 167 & 49 & 1 \\
\hline 74302893 & 212143.60 & -114405.5 & 16.4 & 0.6 & 9011 & 20 & 133 & 12 & 1 \\
\hline 81405246 & 205521.94 & -044141.1 & 16.4 & 0.5 & 24804 & 50 & 452 & 18 & 1 \\
\hline 74402668 & 214634.70 & -095106.0 & 16.4 & 0.8 & 10281 & 37 & 315 & 11 & 1 \\
\hline 74402837 & 214420.85 & -104457.8 & 16.4 & 0.5 & 10820 & 14 & 193 & 8 & 1 \\
\hline 81400846 & $2108 \quad 17.34$ & -035852.3 & 16.4 & 0.5 & 9427 & 5 & 317 & 9 & 1 \\
\hline 74400638 & 214434.04 & -083723.9 & 16.4 & 0.6 & 17950 & 22 & 259 & 17 & 1 \\
\hline 67102743 & 212857.59 & -161804.8 & 16.4 & 0.5 & 18655 & 17 & 320 & 15 & 1 \\
\hline 67201875 & 213448.75 & -145507.4 & 16.4 & 0.5 & 11424 & 17 & 297 & 34 & 1 \\
\hline 81406413 & 205210.94 & -033143.4 & 16.4 & 0.5 & 5823 & 17 & 51 & 15 & 1 \\
\hline 66900782 & 204416.43 & -140903.1 & 16.4 & 0.6 & 8742 & 4 & 110 & 9 & 1 \\
\hline 67100128 & 212920.05 & -135928.8 & 16.4 & 0.6 & 17408 & 13 & 322 & 17 & 1 \\
\hline 88920974 & 215326.20 & -014511.9 & - & 1.0 & 8045 & 17 & 323 & 14 & 1 \\
\hline 88520340 & 204450.30 & +000658.1 & - & 2.0 & 3804 & 16 & 172 & 15 & 1 \\
\hline 88720594 & 211123.79 & +022124.1 & - & 1.9 & 14544 & 37 & 154 & 10 & 3 \\
\hline 88520337 & 204436.89 & +001041.1 & - & 0.7 & 3865 & 7 & 41 & 31 & 3 \\
\hline \multicolumn{10}{|l|}{ Region 3} \\
\hline 68100721 & 004102.46 & -082737.1 & 14.4 & 1.2 & 5960 & 20 & 142 & 14 & 1 \\
\hline 60800316 & 002818.95 & -125946.4 & 15.0 & 0.9 & 6137 & 21 & 73 & 24 & 1 \\
\hline 68101448 & 003208.14 & -110230.3 & 15.0 & 0.8 & 6210 & 18 & 154 & 13 & 1 \\
\hline 68001213 & 001502.00 & -094915.6 & 15.1 & 1.0 & 6718 & 23 & 169 & 7 & 1 \\
\hline 68101358 & 003327.93 & -102349.2 & 15.1 & 0.8 & 5982 & 14 & 195 & 12 & 1 \\
\hline 68101582 & 003000.82 & -122025.9 & 15.1 & 1.1 & 16971 & 5 & 155 & 5 & 2 \\
\hline 61100299 & 012502.07 & -135959.2 & 15.2 & 1.0 & 9894 & 18 & 212 & 11 & 1 \\
\hline 68100166 & 004845.71 & -085207.3 & 15.2 & 0.8 & 4282 & 15 & 168 & 11 & 1 \\
\hline 68301163 & 011359.64 & -081426.4 & 15.2 & 0.7 & 5552 & 16 & 165 & 12 & 1 \\
\hline 61001080 & 010622.24 & -154021.3 & 15.3 & 1.0 & 15911 & 15 & 263 & 21 & 1 \\
\hline 68001229 & 001454.03 & -094827.0 & 15.3 & 1.1 & 6823 & 9 & 118 & 14 & 1 \\
\hline 68100890 & 003918.72 & -093438.6 & 15.4 & 0.8 & 16690 & 24 & 354 & 10 & 2 \\
\hline 68101426 & 003225.81 & -09 3703.4 & 15.4 & 0.7 & 6969 & 11 & 148 & 11 & 1 \\
\hline 68300617 & 012247.56 & -121025.5 & 15.4 & 0.8 & 15021 & 28 & 257 & 15 & 1 \\
\hline 61001389 & 005746.77 & -153406.1 & 15.4 & 0.9 & 16567 & 26 & 341 & 6 & 2 \\
\hline 68100622 & 004227.48 & -090947.5 & 15.4 & 0.7 & 5804 & 3 & 203 & 21 & 3 \\
\hline 68101215 & 003526.55 & -082055.3 & 15.5 & 0.9 & 11214 & 34 & 204 & 9 & 2 \\
\hline 60800542 & 002607.72 & -131711.2 & 15.6 & 0.7 & 7778 & 12 & 223 & 12 & 1 \\
\hline 61002238 & 005622.55 & -164420.3 & 15.6 & 0.8 & 16284 & 28 & 339 & 13 & 3 \\
\hline 68300547 & 012355.82 & -084925.6 & 15.6 & 0.7 & 5253 & 13 & 87 & 11 & 2 \\
\hline 68201452 & 005539.28 & -082915.7 & 15.6 & 1.1 & 4602 & 8 & 178 & 26 & 1 \\
\hline 61001909 & 010621.72 & -161247.6 & 15.7 & 0.9 & 12597 & 21 & 345 & 8 & 2 \\
\hline 68101346 & 003336.91 & -075826.3 & 15.7 & 0.6 & 11191 & 13 & 245 & 15 & 2 \\
\hline
\end{tabular}


Table 5. continued

\begin{tabular}{|c|c|c|c|c|c|c|c|c|c|}
\hline Galaxy & Right Ascension & Declination & $b_{J}$ & $d$ & $v_{\text {hel }}$ & $\operatorname{err}_{\mathrm{v}}$ & $\sigma$ & $\operatorname{err}_{\sigma}$ & $Q_{\text {spec }}$ \\
\hline 60901605 & 003147.85 & -125555.0 & 15.8 & 0.7 & 8030 & 16 & 265 & 13 & 1 \\
\hline 61002004 & 010347.71 & -164834.6 & 15.8 & 0.8 & 11242 & 29 & 276 & 18 & 1 \\
\hline 61100351 & 012338.67 & -134053.1 & 15.8 & 0.9 & 14201 & 26 & 326 & 3 & 2 \\
\hline 68201703 & 005320.82 & -101522.6 & 15.8 & 0.8 & 16356 & 9 & 209 & 12 & 2 \\
\hline 60800452 & 002700.10 & $\begin{array}{lll}-14 & 16 & 10.3\end{array}$ & 15.9 & 0.7 & 16200 & 14 & 203 & 14 & 2 \\
\hline 68201935 & 005105.05 & -083135.7 & 15.9 & 0.8 & 13297 & 28 & 275 & 4 & 1 \\
\hline 68101515 & 003101.35 & -092813.5 & 15.9 & 0.6 & 5543 & 17 & 31 & 23 & 2 \\
\hline 68100388 & 004520.77 & -120350.9 & 15.9 & 0.5 & 5886 & 14 & 87 & 6 & 1 \\
\hline 68101166 & 003602.25 & -09 2315.9 & 16.0 & 0.5 & 6094 & 20 & 44 & 21 & 1 \\
\hline 68201775 & 005243.24 & -113148.3 & 16.0 & 0.7 & 16375 & 20 & 273 & 13 & 1 \\
\hline 68101270 & 003438.49 & -094355.1 & 16.0 & 0.6 & 15877 & 18 & 141 & 18 & 3 \\
\hline 68100879 & 003921.80 & -110624.8 & 16.0 & 0.6 & 15860 & 19 & 199 & 3 & 1 \\
\hline 61102514 & 011652.44 & -165412.4 & 16.1 & 0.6 & 6061 & 8 & 110 & 13 & 2 \\
\hline 68201336 & 005707.98 & -090903.2 & 16.1 & 0.7 & 16614 & 23 & 213 & 15 & 1 \\
\hline 61000670 & 005334.10 & -133741.5 & 16.1 & 0.6 & 16804 & 26 & 408 & 6 & 1 \\
\hline 61100750 & 011438.10 & -141539.3 & 16.1 & 0.6 & 15411 & 29 & 304 & 13 & 1 \\
\hline 68201473 & 005525.46 & -094742.8 & 16.1 & 0.6 & 15060 & 23 & 210 & 17 & 1 \\
\hline 68101018 & 003747.50 & -074343.7 & 16.1 & 0.6 & 3692 & 5 & 6 & 6 & 1 \\
\hline 61100580 & $01 \quad 1830.28$ & -140642.2 & 16.2 & 0.6 & 15384 & 7 & 349 & 18 & 1 \\
\hline 68201394 & 005621.84 & -090242.6 & 16.3 & 0.5 & 8055 & 7 & 133 & 13 & 1 \\
\hline 68100760 & 004038.46 & -100807.2 & 16.3 & 0.5 & 15142 & 7 & 220 & 5 & 3 \\
\hline 68300512 & 012425.53 & -073757.6 & 16.3 & 0.5 & 10191 & 32 & 344 & 3 & 1 \\
\hline 61100364 & $\begin{array}{lll}01 & 23 & 19.43\end{array}$ & -124609.6 & 16.4 & 0.5 & 10262 & 24 & 218 & 3 & 1 \\
\hline 61101581 & 011808.13 & -140733.0 & - & - & 14708 & 3 & 246 & 12 & 1 \\
\hline 61101582 & $01 \quad 1806.14$ & -140733.6 & - & - & 16458 & 10 & 236 & 18 & 1 \\
\hline 61101583 & 011752.50 & -140906.4 & - & - & 15292 & 19 & 224 & 8 & 1 \\
\hline 61101584 & $01 \quad 1752.46$ & -141409.7 & - & - & 20954 & 5 & 326 & 11 & 1 \\
\hline 61001081 & 010629.38 & $-1541 \quad 12.2$ & - & - & 17451 & 28 & 454 & 10 & 2 \\
\hline 61001082 & 010621.22 & -154117.3 & - & - & 16530 & 29 & 170 & 13 & 2 \\
\hline 61001084 & 010625.64 & -154215.1 & - & - & 16913 & 12 & 228 & 8 & 2 \\
\hline 61001085 & 010633.34 & -154650.8 & - & - & 16300 & 10 & 158 & 17 & 3 \\
\hline
\end{tabular}
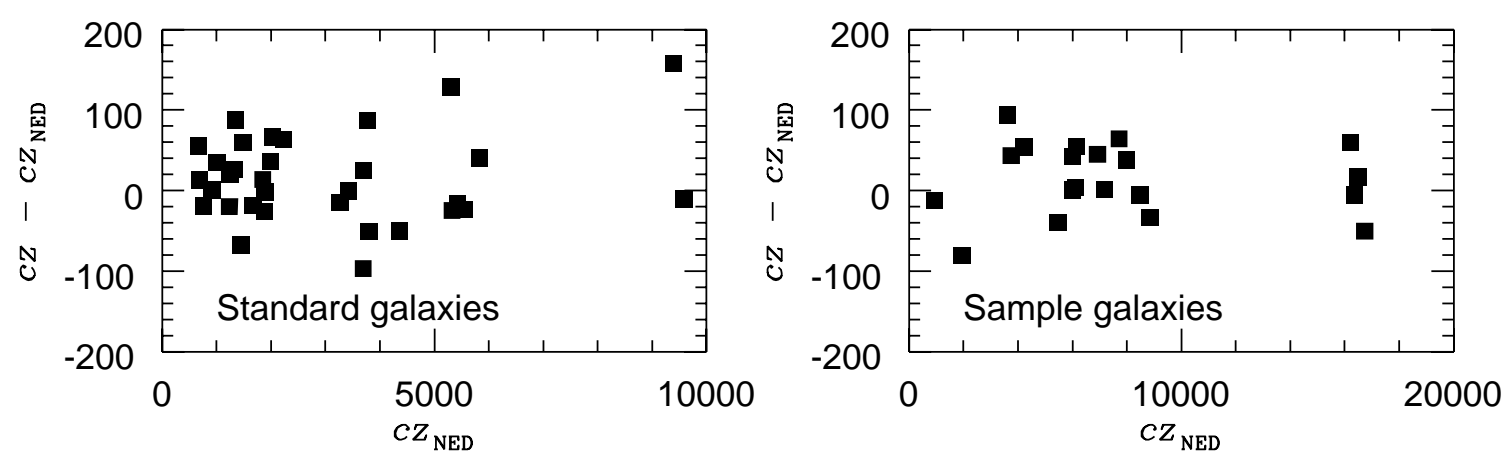

Fig. 10. Comparison of results for heliocentric radial velocity with published data for 32 standard galaxies and 20 sample galaxies 
Table 6. Spectroscopic parameters for the standard galaxies

\begin{tabular}{lrrrrrrr}
\hline Galaxy & Right Ascension & Declination & $v_{\text {hel }}$ & err $_{\mathrm{v}}$ & $\sigma$ & err $_{\sigma}$ & $Q_{\text {spec }}$ \\
\hline NGC 5831 & 150134 & +012454 & 1648 & 19 & 139 & 3 & 1 \\
NGC 5845 & 150328 & +014936 & 1383 & 15 & 196 & 2 & 1 \\
NGC 5846A & 150356 & +014712 & 1688 & 18 & 232 & 3 & 1 \\
NGC 6020 & 155500 & +223300 & 4307 & 16 & 173 & 15 & 1 \\
NGC 6051 & 160248 & +240354 & 9578 & 17 & 347 & 13 & 2 \\
NGC 6086 & 161036 & +293654 & 9549 & 25 & 313 & 15 & 1 \\
NGC 7619 & 231743 & +075600 & 3753 & 14 & 269 & 14 & 1 \\
NGC 7626 & 231810 & +075636 & 3431 & 15 & 313 & 8 & 1 \\
NGC 227 & 004303 & -014818 & 5434 & 16 & 243 & 15 & 1 \\
IC 1696 & 012219 & -015242 & 5860 & 11 & 166 & 8 & 2 \\
NGC 533 & 012257 & +013000 & 5521 & 13 & 224 & 20 & 1 \\
NGC 636 & 013636 & -074554 & 1861 & 19 & 155 & 3 & 1 \\
NGC 7468 & 230030 & +162000 & 2094 & 22 & 137 & 40 & 2 \\
NGC 7751 & 234424 & +063500 & 3247 & 10 & 123 & 3 & 2 \\
NGC 541 & 012312 & -013800 & 5401 & 20 & 186 & 14 & 1 \\
NGC 545 & 012324 & -013600 & 5296 & 18 & 203 & 14 & 2 \\
NGC 1550 & 041700 & +021800 & 3714 & 23 & 327 & 15 & 1 \\
NGC 1587 & 042806 & +003300 & 3597 & 22 & 239 & 16 & 1 \\
\hline
\end{tabular}

9. Estimated internal error in $\sigma$ (in $\left.\mathrm{km} \mathrm{s}^{-1}\right)$.

10. Quality rating $Q_{\text {spec }}$ for the spectrum.

In Table 5 , the galaxies are ordered by magnitude, with a few exceptions. The galaxies CGCG2158 (in Region 1) and NGC 7010 (in Region 2) were added for completeness. In Region 1, the galaxy denoted $87101109 \mathrm{E}$ is the elliptical galaxy close to 87101109 , which was found to be a spiral. Also included are some galaxies from plates which are not in the final APM catalog: two galaxies from plate 728 in Region 1, and four galaxies (from plates 885, 887, and 889 ) in Region 2 (no magnitudes are given for these galaxies, since the magnitude zero-pointing and plate-matching calibration was not done for these plates). In Region 3, there are eight extra galaxies: a group near 61101581 and a group near 61101081 , for which spectra had been obtained, and which were included in the sample.

The results for the spectroscopic parameters of the standard galaxies and Coma galaxies are presented in Tables 6 and 7 respectively. The columns are as in Table 5, except that magnitudes and major-axis diameters are not given. The names of the galaxies in Coma are taken from the Dressler numbers as catalogued by Dressler (1980).

\section{Internal and external comparisons}

\subsection{Internal comparisons}

The reliability of the determinations of the spectroscopic parameters was checked by comparing the results from re-

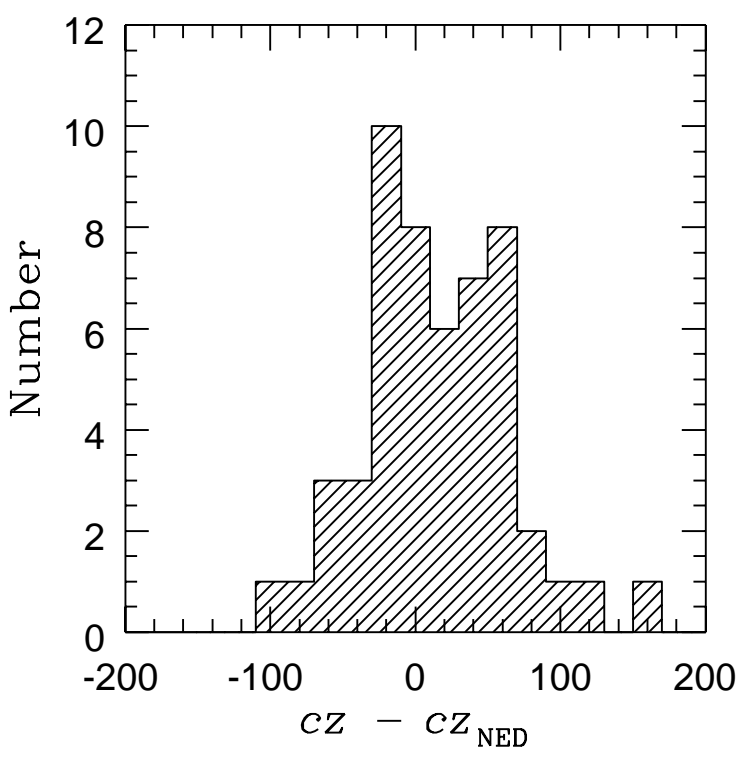

Fig. 11. Distribution of differences between measured redshifts and the redshifts given by NED (literature redshifts), for 52 standard galaxies and sample galaxies

peat observations of the same galaxy. There are 30 galaxies of which two spectra were obtained for each, and for these galaxies the differences in parameters were found. 
Table 7. Spectroscopic parameters for the Coma galaxies

\begin{tabular}{|c|c|c|c|c|c|c|c|}
\hline Galaxy & Right Ascension & Declination & $v_{\text {hel }}$ & $\operatorname{err}_{\mathrm{v}}$ & $\sigma$ & $\operatorname{err}_{\sigma}$ & $Q_{\text {spec }}$ \\
\hline Coma 31 & 125459.0 & +274600 & 7369 & 15 & 256 & 14 & 1 \\
\hline Coma 46 & 125507.5 & +275255 & 6074 & 12 & 210 & 13 & 1 \\
\hline Coma 49 & 125929.9 & +275336 & 7893 & 15 & 267 & 11 & 1 \\
\hline Coma 69 & 125643.5 & +280316 & 7072 & 29 & 265 & 39 & 3 \\
\hline Coma 70 & 125642.8 & +280221 & 6336 & 7 & 187 & 27 & 2 \\
\hline Coma 72 & 125627.2 & +280333 & 5656 & 14 & 199 & 4 & 2 \\
\hline Coma 87 & 125705.9 & +280345 & 7825 & 6 & 66 & 37 & 3 \\
\hline Coma 88 & 125704.5 & +280709 & 6790 & 19 & 236 & 16 & 3 \\
\hline Coma 103 & 125706.0 & +280913 & 4744 & 8 & 205 & 4 & 1 \\
\hline Coma 105 & 125658.7 & +281059 & 5104 & 25 & 170 & 10 & 2 \\
\hline Coma 106 & 125658.1 & +281005 & 5087 & 8 & 199 & 11 & 2 \\
\hline Coma 107 & 125655.6 & +280925 & 6452 & 9 & 138 & 1 & 3 \\
\hline Coma 118 & 125815.2 & +281142 & 7552 & 28 & 141 & 8 & 1 \\
\hline Coma 124 & 125719.8 & +281102 & 6678 & 4 & 236 & 6 & 1 \\
\hline Coma 125 & 125717.9 & +281147 & 6900 & 3 & 174 & 5 & 2 \\
\hline Coma 129 & 125711.1 & +281353 & 7223 & 11 & 266 & 11 & 3 \\
\hline Coma 130 & 125709.6 & +281309 & 7209 & 8 & 234 & 1 & 2 \\
\hline Coma 133 & 125650.6 & +281433 & 4859 & 7 & 257 & 5 & 1 \\
\hline Coma 134 & 125639.4 & +281351 & 6978 & 6 & 184 & 1 & 2 \\
\hline Coma 135 & 125635.7 & +281423 & 8286 & 33 & 137 & 14 & 2 \\
\hline Coma 136 & 125630.6 & +281412 & 5682 & 14 & 266 & 9 & 2 \\
\hline Coma 143 & 125830.1 & +281642 & 4990 & 15 & 217 & 13 & 1 \\
\hline Coma 148 & 125743.7 & +281454 & 6360 & 22 & 431 & 20 & 1 \\
\hline Coma 150 & 125742.0 & +281633 & 7241 & 5 & 202 & 15 & 3 \\
\hline Coma 151 & 125740.0 & +281532 & 6350 & 5 & 157 & 21 & 2 \\
\hline Coma 153 & 125719.2 & +281557 & 6912 & 90 & 215 & 9 & 1 \\
\hline Coma 159 & 125648.4 & +281454 & 6881 & 20 & 252 & 4 & 1 \\
\hline Coma 160 & 125641.0 & +281558 & 7672 & 14 & 153 & 13 & 1 \\
\hline Coma 168 & 125824.5 & +282140 & 7088 & 18 & 226 & 23 & 3 \\
\hline Coma 172 & 125750.3 & +281845 & 5827 & 9 & 211 & 15 & 3 \\
\hline Coma 179 & 125653.1 & +282113 & 4638 & 16 & 234 & 15 & 1 \\
\hline Coma 180 & 125649.3 & +282053 & 7780 & 11 & 104 & 6 & 3 \\
\hline Coma 193 & 125730.8 & +282400 & 7501 & 20 & 126 & 12 & 2 \\
\hline Coma 194 & 125639.3 & +282347 & 7980 & 2 & 285 & 2 & 2 \\
\hline Coma 206 & 125750.9 & +282817 & 8507 & 17 & 268 & 15 & 1 \\
\hline Coma 207 & 125743.8 & +282631 & 6779 & 16 & 82 & 12 & 2 \\
\hline Coma 217 & 125732.8 & +283108 & 6803 & 9 & 194 & 4 & 3 \\
\hline Coma 232 & 125803.8 & +283655 & 6012 & 11 & 163 & 13 & 1 \\
\hline Coma 239 & 125507.0 & +284428 & 6322 & 3 & 205 & 1 & 1 \\
\hline Coma 240 & 125509.0 & +284506 & 6834 & 15 & 246 & 2 & 1 \\
\hline
\end{tabular}

\subsubsection{Redshifts}

Internal comparisons of radial velocities are shown in Fig. 6 for 30 galaxies with two spectra each. The order $\left(c z_{1}\right.$ and $\left.c z_{2}\right)$ in which the two results have been considered is arbitrary. The rms scatter is $\Delta c z_{\text {hel }}=40.7 \mathrm{~km} \mathrm{~s}^{-1}$ for the comparisons of repeat observations.

\subsubsection{Velocity dispersions}

In Fig. 7, internal comparisons of velocity dispersions are shown for 29 galaxies with two spectra each. An estimate of the accuracy is the rms scatter of $\left|\log \sigma_{1}-\log \sigma_{2}\right|$, which is 0.045 . In Fig. 8, the errors in repeat measurements of $\sigma$ are also shown as a function of $S / N$ per $\AA$. It is clear from the plot that the repeatability depends on the $S / N$ of the spectrum. For a $S / N$ of $40-50$, velocity dispersions can be determined to within $4-5 \%$ (from $\Delta \sigma / \sigma$ ) and for a $S / N$ of $\sim 20$ (which is the case for most of the galaxies in the sample) the accuracy is about $8-10 \%$.

Figure 9 is a histogram of the fractional errors in $\sigma$ for 29 repeat observations. The rms scatter in $\Delta \sigma / \sigma$ is 0.103 , which means that $\sigma$ can be determined to $10 \%$ accuracy. The galaxies for which there are repeat measurements are typical of those in the whole sample, so these estimated errors are representative of the true errors. 

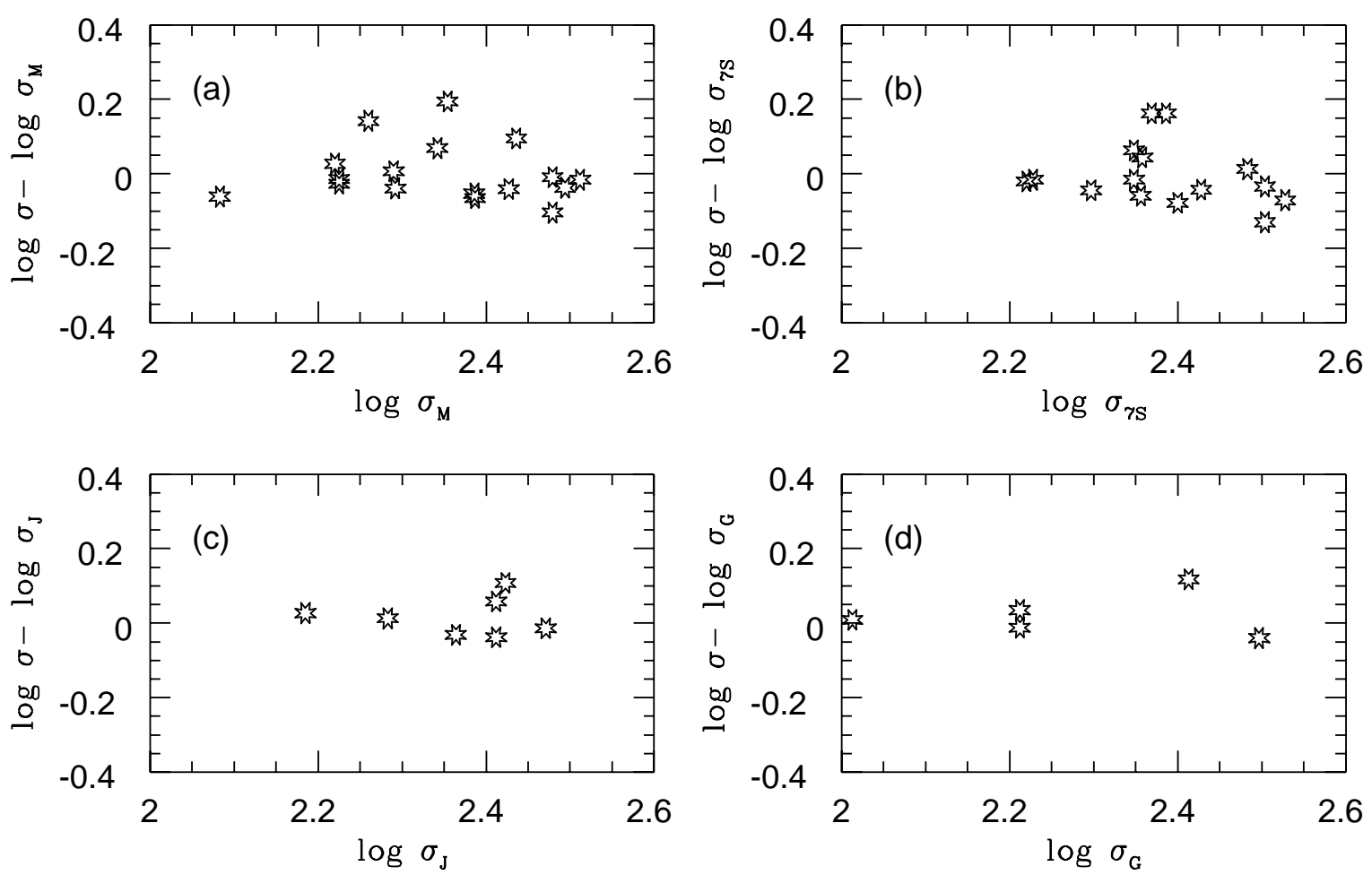

Fig. 12. Comparison of the results for velocity dispersions of standard galaxies with values from the literature: a) McElroy (1995); b) Davies et al. (1987); c) Jørgensen et al. (1995); d) González (1993)

\subsection{External comparisons}

\subsubsection{Redshifts}

Results for redshifts were compared with values from $\mathrm{NED}^{3}$ (Madore et al. 1992) and from the ZCAT compilation of redshifts (Huchra et al. 1992), and the differences were found to be relatively small. Redshift comparisons were possible for 32 standard galaxies and 20 sample galaxies. (Redshifts were available for only a small fraction of the galaxies in the sample regions, since the South Equatorial Strip had not been well studied before.)

The determinations of radial velocity for these two sets of galaxies were compared with redshifts from the literature, and the results are shown in Fig. 10. For both data sets the agreement is good. For all 52 galaxies for which literature redshifts were available, the distribution of differences between the measured redshifts and the literature values is shown in the histogram of Fig. 11. The values of the mean absolute difference $|c z-c z \mathrm{NED}|$ are $42.4 \mathrm{~km} \mathrm{~s}^{-1}$ and $37.3 \mathrm{~km} \mathrm{~s}^{-1}$ for the standard galaxy and sample galaxy comparisons respectively; the rms differences are $56.0 \mathrm{~km} \mathrm{~s}^{-1}$ and $45.8 \mathrm{~km} \mathrm{~s}^{-1}$. This shows that the redshifts are of adequate reliability.

\footnotetext{
${ }^{3}$ NED, the NASA/IPAC Extragalactic Database, is operated by the Jet Propulsion Laboratory, California Institute of Technology under contract with the National Aeronautics and Space Administration.
}

7.2.2. Velocity dispersions

In order to make sure that the values of velocity dispersions determined here are scaled to the standard system, the results for the velocity dispersions of standard galaxies were compared with published values. Our data set overlapped with the following sets: (i) the compilation of McElroy (1995) (17 galaxies in common); (ii) Davies et al. (1987) (7S) (15 galaxies in common); (iii) Jørgensen et al. (1995) (7 galaxies in common); and (iv) González (1993) (5 galaxies in common).

The data set of Davies et al. (the 7S data) defines a good standard system since it is frequently used for comparisons. The comparison with the results of Davies et al. is shown in Fig. 12 together with comparisons with data from the other sources. The difference between the values from this study and the literature data, $\Delta \log \sigma=\log \sigma$ (this study) $-\log \sigma$ (literature), are shown plotted against $\log \sigma$ (literature). There do not appear to be any offsets or systematic differences relative to the velocity dispersions of the four data sets. The rms scatter of $\Delta \log \sigma$ is $0.066,0.111,0.036$, and 0.021 for the plots in (a), (b), (c), and (d) respectively. The agreement is best for comparisons with González (1993) and Jørgensen et al. (1995), which are the most recent data sets, although the numbers of galaxies in common are smaller. 


\section{Summary}

We have obtained new radial velocities and central velocity dispersions for $179 \mathrm{E}$ and S0 galaxies in three selected directions in the APM South Equatorial Strip, as well as for 40 galaxies in the Coma cluster. Observations were made with the $2.4 \mathrm{~m}$ and $1.3 \mathrm{~m}$ telescopes of the MDM Observatory on Kitt Peak, Arizona, using the Mark III spectrograph, and at the $4.4 \mathrm{~m} \mathrm{MMT}$, using the Red Channel. The spectra have a mean $S / N$ per $\AA$ of 23 .

Radial velocities and central velocity dispersions have been determined by the Fourier cross-correlation method. The velocity dispersions have been corrected for the effect of the aperture size and for the galaxy's effective radius. We find that the typical uncertainties on the derived parameters are $\pm 40 \mathrm{~km} \mathrm{~s}^{-1}$ in $c z$ and \pm 0.045 in $\log \sigma$. In comparisons with literature data, no offsets or systematic differences are seen. The accuracy of $8-10 \%$ in the derived velocity dispersions is high enough for the values to be used in the application of Fundamental Plane analysis. The results given here have been used together with photometric data (Müller et al. 2000) to derive Fundamental Plane distances to the sample galaxies in order to determine their peculiar velocities and thereby investigate the reality of large-scale streaming motion; results have been reported in Müller et al. (1998).

Acknowledgements. GW wishes to acknowledge partial support from the Alexander von Humboldt Foundation during a visit to the Ruhr-Universität Bochum, and also from NSF grant AST93-47714. KM acknowledges financial support from a Dartmouth Fellowship and from a Research Studentship at the European Southern Observatory in Munich.

\section{References}

Abt H.A., Biggs E.S., 1972, Bibliography of Stellar Radial Velocities (KPNO). Latham Process Corp., New York

Baggley G., 1996, Ph.D. Thesis, Oxford University

Barbier-Brossat M., Petit M., 1987, Catalogue Bibliographique de Vitesses Radiales Stellaires

Dalle Ore C., Faber S.M., González J.J., Stoughton R., 1991, ApJ 366, 38

Davies R.L., Birkinshaw M., 1988, ApJS 68, 409

Davies R.L., Burstein D., Dressler A., et al., 1987, ApJS 64, 581

Davies R.L., Sadler E.M., Peletier R.F., 1993, MNRAS
262,650

Dressler A., 1979, ApJ 231, 659

Dressler A., 1980, ApJS 42, 565

Evans D.S., 1970, Catalogue de Vitesses Radiales Stellaires Moyennes, Observatoire de Marseille

Feldman H.A., Watkins R., 1994, ApJ 430, L17

Franx M., Illingworth G.D., Heckman T., 1989, ApJ 344, 613

Giovanelli R., Haynes M.P., Wegner G., et al., 1996, ApJ 464, L99

Giovanelli R., Haynes M.P., Freudling W., et al., 1998a, ApJ 505, L91

Giovanelli R., Haynes M.P., Salzer J., et al., 1998b, AJ 116, 2632

González J.J., 1993, Ph.D. Thesis, University of California at Santa Cruz

Huchra J.P., Geller M.J., Clemens C., Tokarz S., Michel A., 1992, Bull. CDS 41, 31

Jørgensen I., Franx M., Kjærgaard P., 1993, ApJ 411, 34

Jørgensen I., Franx M., Kjærgaard P., 1995, MNRAS 276, 1341

Kogut A., Lineweaver C., Smoot G.F., et al., 1993, ApJ 419, 1

Lauer T.R., Postman M., 1994, ApJ 425, 418

Lucey J.R., Bower R.G., Ellis R.S., 1991, MNRAS 249, 755

Madore B.F., Helou G., Corwin Jr. H.G., et al., 1992, in: Worrall D.M., Biemesderfer C., Barnes J. (eds.) Astronomical Data Analysis Software and Systems I, ASP Conf. Ser. 25, Astron. Soc. Pac., San Francisco, p. 47

McElroy D.B., 1995, ApJS 100, 105

Müller K.R., 1997, Ph.D. Thesis, Dartmouth College

Müller K.R., Freudling W., Watkins R., Wegner G., 1998, ApJ 507, L105

Müller K.R., Freudling W., Wegner G., 2000 (in preparation)

Raychaudhury S., Lynden-Bell D., 2000, A Catalogue of Galaxies in the Southern Equatorial Strip. Cambridge University Press, Cambridge UK

Riess A.G., Press W.H., Kirschner R.P., 1995, ApJ 445, L91

Sargent W.L.W., Schechter P.L., Boksenberg A., Shortridge K., 1977, ApJ 212, 326

Scodeggio M., Giovanelli R., Haynes M.P., 1997, AJ 113, 101

Strauss M.A., Willick J.A., 1995, Phys. Rep. 261, 271

Strauss M.A., Cen R.Y., Ostricker J.P., et al., 1995, ApJ 444, 507

Strom K.M., 1979, Standard Stars for Intensified Image Dissector Scanner Observations, Kitt Peak National Observatory Booklet

Tody D., 1986, Proc. SPIE 627, 733

Tonry J.L., Davis M., 1979, AJ 84, 1511

Wilson R.E., 1953, General Catalogue of Stellar Radial Velocities, Carnegie Institute of Washington Publication 601, Washington DC

Zabludoff A.I., Huchra J.P., Geller M.J., 1993, AJ 105, 788 